\title{
The Structure of Hilbert Flag Varieties
}

\author{
Dedicated to the memory of our father
}

By

\author{
Gerard F. Helminck* and Aloysius G. Helminck**
}

\begin{abstract}
In this paper we present a geometric realization of infinite dimensional analogues of the finite dimensional representations of the general linear group. This requires a detailed analysis of the structure of the flag varieties involved and the line bundles over them. In general the action of the restricted linear group can not be lifted to the line bundles and thus leads to central extensions of this group. It is determined exactly when these extensions are non-trivial. These representations are of importance in quantum field theory and in the framework of integrable systems. As an application, it is shown how the flag varieties occur in the latter context.
\end{abstract}

\section{§1. Introduction}

Let $H$ be a complex Hilbert space. If $H$ is finite dimensional, then it is a classical result that the finite dimensional irreducible representations of the general linear group $G L(H)$ can be realized geometrically as the natural action of the group $G L(H)$ on the space of global holomorphic sections of a holomorphic line bundle over a space of flags in $H$. By choosing a basis of $H$, one can identify this space of holomorphic sections with a space of holomorphic functions on $G L(H)$ that are certain polynomial expressions in minors of the matrices corresponding to the elements of $G L(H)$. Infinite dimensional analogues of some of these representations occur in quantum field theory, see e.g. [5]. Infinite dimensional Grassmann manifolds play an important role in the framework of integrable systems. The first person to realize this was Sato, see [24].

In this paper we will give an infinite dimensional analogue of all these representations. Thereto we take a separable Hilbert space $H$. In $H$ we consider a collection of flags that generalizes the Grassmanian from chapter 7 in [23]. This flag variety carries a natural Hilbert space structure and there

Communicated by T. Miwa, January 18, 1993.

1991 Mathematics Subject Classification: 22E65, 14M15, 35Q58, 43A80, 17 B65.

* Department of Mathematics, Universiteit Twente, Enschede, The Netherlands

** Department of Mathematics, North Carolina State University, Raleigh, NC 27695, USA 
exist line bundles over it that are similar to the finite dimensional ones. This includes the determinant bundle and its dual from [23]. In the "dominant" case the space of global holomorphic sections of such a line bundle turns out to be non-trivial. However, the action of the analogue of the general linear group can, in general, not be lifted to the line bundle under consideration and one has to pass to a central extension of this group. Besides of the introduction, this paper consists of three sections. In the first section we give the definition of the flag variety $\mathfrak{F}$ and we treat some properties of $\mathfrak{F}$. The second section is devoted to the construction of the holomorphic line bundles, to a description of the corresponding central extensions and to the analysis of the space of global holomorphic sections. As an application, we show in the final section what role the geometry plays in the context of some integrable systems. A more detailed description of the content of the different sections is as follows.

The first subsection of section 2 discusses the type of flags in $H$ that will be considered. Here the model for the size of the flags is the basic flag $F^{(0)}$ corresponding to a finite orthogonal decomposition of $H$. The flag variety $\mathfrak{F}$ is a homogeneous space for a certain unitary group $U_{\text {res }}(H)$. As in the finite dimensional case it is convenient to see $\mathfrak{F}$ also as a homogeneous space for a larger group of automorphisms of $H$, namely $G L_{\text {res }}(H)$. This is the analogue of the general linear group in this framework. Analogously to the finite dimensional case the group $U_{\text {res }}(H)$ is the unitary component in the polar decomposition of $G L_{\text {res }}(H)$. In the second subsection we give an explicit description of the manifold structure on $\mathfrak{F}$ and we discuss decompositions of certain open subsets of $G L_{\text {res }}(H)$. A first difference with the finite dimensional situation appears at the description of the connected components of $F$ in the third subsection. The fourth subsection contains the technical prerequisites for the construction of the line bundles. First we choose a suitable orthonormal basis of $H$, we order its index set conveniently and we introduce the Weyl group $W$ of $G L_{\text {res }}(H)$. Next we show that the charts around the points in the $W$-orbit through $F^{(0)}$ cover $\mathfrak{F}$. By using this covering one obtains a stratification of $\mathfrak{F}$ into parts that are all homeomorphic to a Hilbert space. On the group level this gives you the Birkhoff decomposition for $G L_{\text {res }}(H)$.

Let $\mathfrak{F}^{(0)}$ be the connected component of $\mathfrak{F}$ containing $F^{(0)}$. From the foregoing results one deduces that $\mathfrak{F}^{(0)}$ is a homogeneous space for a Banach Lie group (5 that permits you to take suitable minors. As a group the group $\left(5\right.$ is a subgroup of $G L_{\text {res }}(H)$, but its topology is stronger than the one induced by $G L_{\text {res }}(H)$. The description of $(5$ and its topology can be found in the first subsection of section 3. There we introduce also the maximal torus $T\left(.1^{\prime}\right)$ of (5 and its group of analytic characters $\hat{T}$. In the second subsection we introduce a dense tower of finite dimensional flag varieties in $\mathfrak{F}^{(0)}$. The next subsection 
shows how you can associate to certain elements $\psi_{\underline{h}}, k \in \mathbf{Z}^{m}$, of $\hat{T}$ a holomorphic line bundle $L(\underline{k})$ over $\mathfrak{F}^{(0)}$. Further it is shown there that, if one tries to lift the action of the connected component $G L_{\text {res }}^{(0)}(H)$ of $G L_{\text {res }}(H)$, one might meet obstructions and that one can only lift the action of a central extension of $G L_{\text {res }}^{(0)}(H)$. The natural question that comes up then is "how essential is this extension". This question is treated fully in the next subsection. Then one has come to the final subsection of this section. There we determine, when $L(\underline{k})$ has global non-trivial sections and we show that the action of $(5$ on this space defines an irreducible (5-module of highest weight $\psi_{\underline{k}}$.

Section 4 is an illustration of the fact that the geometry of the foregoing sections plays a role in the theory of integrable systems. The system we will consider is the multicomponent KP-hierarchy. The first subsection describes the flows in $G L_{\text {res }}(H)$ corresponding to this system. The algebraic framework for this system of equations is given in the second subsection. In the final subsection of this paper we indicate how the flag varieties form the starting point of the construction of solutions to the equations of the multicomponent KP-hierarchy and the modified versions of the KP-hierarchy.

We would like to thank the referee for bringing to our attention papers by Faltings [10] and Kashiwara [19], who give an algebraic geometrical approach to infinite dimensional flag varieties.

\section{§2. Properties of Hilbert Flag Varieties}

2.1. The flag variety. Let $H$ be a separable complex Hilbert space with inner product $\langle\cdot, \cdot\rangle$. We will consider certain finite chains of subspaces in $H$ and we will call them flags as in the finite dimensional case. First one has to specify the "size" of the components of the flag. Therefore we start with an orthogonal decomposition of $H$,

$$
H=H_{1} \oplus \cdots \oplus H_{m}, \quad \text { where } \quad H_{i} \perp H_{j} \text { for } i \neq j .
$$

We assume that $m_{i}=\operatorname{dim}\left(H_{i}\right)$ satisfies $1 \leq m_{i} \leq \propto$. An example of a decomposition occurring in quantum field theory is the one corresponding to the positive and negative spectrum of the Dirac operator, see [5] and [21]. In the context of integrable systems we have:

Example 2.1.1. Let $(\cdot, \cdot)$ be the standard inner product on $\mathbf{C}^{r}$. The Grassmann manifold $\operatorname{Gr}(H)$ that is crucial at the construction of solutions of KP-type hierarchies in [23], [13] and [25] corresponds to the case that $H$ is the space of power series

$$
\begin{aligned}
& H=L^{2}\left(S^{1}, \mathbf{C}^{r}\right)=\left\{\sum_{n \in \mathbf{Z}} a_{n} z^{n}, a_{n} \in \mathbf{C}^{r}, \sum_{n \in \mathbf{Z}}\left(a_{n}, a_{n}\right)<x\right\}, \\
& H_{1}=\left\{\sum_{n \geq 0} a_{n} z^{n} \in H\right\} \text { and } H_{2}=\left\{\sum_{n<0} a_{n} z^{n} \in H\right\} .
\end{aligned}
$$


If one takes $r=1$ and $k$ and $l$ in $\mathbf{Z}$ with $k>l$, then the basic manifold corresponding to the $(k, l)$-modified $\mathrm{KP}$-hierarchy is the flag variety corresponding to $H=L^{2}\left(S^{1}, \mathbf{C}\right)=H_{1} \oplus H_{2} \oplus H_{3}$, with

$$
H_{1}=\left\{\sum_{n \geq k} a_{n} z^{n} \in H\right\}, H_{2}=\left\{\sum_{n \geq l}^{k-1} a_{n} z^{n} \in H\right\} \quad \text { and } \quad H_{3}=\left\{\sum_{n<l} a_{n} z^{n} \in H\right\} .
$$

This correspondence is described in the fourth section.

Let $p_{i}, 1 \leq i \leq m$, be the orthogonal projection of $H$ onto $H_{i}$. Then we will use throughout this paper the following

Notation 2.1.2. If $g$ belongs to $\mathscr{B}(H)$, the space of bounded linear operators from $H$ to $H$, then $g=\left(g_{i j}\right), 1 \leq i \leq m$ and $1 \leq j \leq m$ denotes the decomposition of $g$ w.r.t. the $\left\{H_{i} \mid 1 \leq i \leq m\right\}$. That is to say $g_{i j}=p_{i} \circ g \mid H_{j}$.

Remark 2.1.3. Let $K_{i}, i=1,2$, be Hilbert spaces with inner products $\langle,\rangle_{i}, i=1,2$. If $A$ belongs to $\mathscr{B}\left(K_{1}, K_{2}\right)$, the space of bounded linear operators from $K_{1}$ to $K_{2}$, then its adjoint $A^{*}: K_{2} \rightarrow K_{1}$ is defined by

$$
\left\langle A\left(k_{1}\right), k_{2}\right\rangle_{2}=\left\langle k_{1}, A^{*}\left(k_{2}\right)\right\rangle_{1} .
$$

If $g=\left(g_{i j}\right)$ as in notation 2.1.2, then we have for its adjoint $g^{*}$ the decomposition $\left(g^{*}\right)_{i j}=\left(g_{j i}\right)^{*}$, for all $i$ and $j$.

2.1.4. To the decomposition (1) we associate the basic flag $F^{(0)}$ given by

$$
0 \subset H_{1} \subset \cdots \subset \bigoplus_{j=1}^{r} H_{j} \subset \cdots \subset H
$$

Now we consider in $H$ flags $F=\{F(0), \ldots, F(m)\}$, that is to say chains of closed subspaces of $H$,

$$
\{0\}=F(0) \subset F(1) \subset \cdots \subset F(m)=H,
$$

that are of the same "size" as the basic flag $F^{(0)}$, i.e. for all $i, 1 \leq i \leq m$,

$$
\operatorname{dim}(F(i) / F(i-1))=\operatorname{dim}\left(H_{i}\right) .
$$

To such a flag $F$ is associated an orthogonal decomposition of $H$,

$$
H=F_{1} \oplus \cdots \oplus F_{m} \text {, where } F_{i}=F(i) \cap F(i-1)^{\perp} .
$$

We will denote such a flag $F$ by $F=\{F(0), \ldots, F(m)\}$ as well as $F=\left\{F_{1}, \ldots F_{m}\right\}$.

The class of flags one obtains in this way is still too wide and we will require that our flags do not differ too much from the basic flag. One can express this "nearness" in various ways. Our choice is a natural generalization of that used in [23] for the Grassmann manifold. However, a lot of the constructions given here for that case can be carried out with some minor 
adjustments also for other choices. We start by introducing notations for some spaces of compact operators that occur in the sequel.

Notation 2.1.5. If $K_{1}$ and $K_{2}$ are Hilbert spaces, then we denote the space of Hilbert-Schmidt operators from $K_{1}$ to $K_{2}$ by $\mathscr{H} \mathscr{S}\left(K_{1}, K_{2}\right)$ and the Hilbert-Schmidt norm by $\|\cdot\|_{\mathscr{H} \mathscr{S}}$. We will write $\mathscr{N}\left(K_{1}, K_{2}\right)$ for the space of nuclear operators from $K_{1}$ to $K_{2}$ and the trace norm on it will be denoted by $\|\cdot\|_{\mathcal{N}}$. The space $\mathscr{C}\left(K_{1}, K_{2}\right)$ of compact operators from $K_{1}$ to $K_{2}$ will be assumed to have been equipped with the operator norm. Then we have the following chain of continuous inclusions:

$$
\mathscr{N}\left(K_{1}, K_{2}\right) \subset \mathscr{H} \mathscr{S}\left(K_{1}, K_{2}\right) \subset \mathscr{C}\left(K_{1}, K_{2}\right) .
$$

In each of these spaces the collection of finite dimensional operators $\mathscr{F}\left(K_{1}, K_{2}\right)$ lies dense. If $K_{2}$ is equal to $K_{1}$, then we simply write $\mathscr{F}\left(K_{1}\right), \mathscr{N}\left(K_{1}\right), \mathscr{H} \mathscr{S}\left(K_{1}\right)$ and $\mathscr{C}\left(K_{1}\right)$ for respectively $\mathscr{F}\left(K_{1}, K_{1}\right), \mathcal{N}\left(K_{1}, K_{1}\right), \mathscr{H} \mathscr{S}\left(K_{1}, K_{1}\right)$ and $\mathscr{C}\left(K_{1}, K_{1}\right)$.

Definition 2.1.6. Let $\mathfrak{F}$ be the collection of flags $F=\left\{F_{1}, \ldots, F_{m}\right\}$, satisfying $\operatorname{dim}\left(F_{i}\right)=\operatorname{dim}\left(H_{i}\right)$, and for all $i$ and $j$ with $j \neq i$, the orthogonal projection $p_{j}: F_{i} \rightarrow H_{j}$ is a Hilbert-Schmidt operator. We call $\mathfrak{F}$ the flag variety corresponding to the decomposition (1).

Remark 2.1.7. If only one $m_{i}$ is infinite, then the Hilbert-Schmidt condition is superfluous. E.g. the space of flags with $m_{i}<\infty$ for all $i<m$, plays a role in [2] at the construction of irreducible representations of the Hilbert Lie group $U(\mathfrak{H})_{2}$. This is the unitary part of the group of invertible transformations of the form "identity + a Hilbert-Schmidt operator".

Remark 2.1.8. Instead of the condition $p_{j}: F_{i} \rightarrow H_{j}, i \neq j$, belongs to $\mathscr{H} \mathscr{S}\left(F_{i}, H_{j}\right)$, one could also consider flags such that this map belongs to $\mathscr{N}\left(F_{i}, H_{j}\right)$ or $\mathscr{C}\left(F_{i}, H_{j}\right)$. The flag varieties one obtains in this way we denote by $\mathfrak{F}(\mathscr{N})$ respectively $\mathfrak{F}(\mathscr{C})$. A more asymmetric condition is considered in [14] where it is required that merely for $i<j$ the projection $p_{i}: F_{j} \rightarrow H_{i}$ is Hilbert-Schmidt. In this way we get the flag manifold $\mathfrak{F}(\mathscr{B})$. Because of the inclusions mentioned above, we have a chain of injections

$$
\mathfrak{F}(\mathscr{N}) \subset \mathfrak{F} \subset \mathfrak{F}(\mathscr{C}) \subset \mathfrak{F}(\mathscr{B}) .
$$

For $m=2$ more general versions of flag spaces are considered in [9].

Remark 2.1.9. In [8], they associate a Banach Grassmann manifold to each Banach Jordan pair. It would be interesting to see if, and if so, how the flag varieties introduced here fit into their framework.

2.1.10. The space $\mathfrak{F}$ is a natural generalization of the Grassmann manifold introduced in section 7.1 of [23]. The flag variety $\mathfrak{F}$ is a homogeneous space 
for an analogue adapted to this situation, of the general linear group. The Banach structure of this group follows directly from that of its Lie algebra. Therefore we start with the analogue of the Lie algebra of the general linear group.

Definition 2.1.11. A restricted endomorphism of $H$ is a $u=\left(u_{i j}\right)$ in $\mathscr{B}(H)$ such that $u_{i j}$ is a Hilbert-Schmidt operator for all $i \neq j$. We denote the space of all restricted endomorphisms of $H$ by $\mathscr{B}_{\text {res }}(H)$.

For all $i$ and $j$, we extend the elements of $\mathscr{H} \mathscr{S}\left(H_{i}, H_{j}\right)$ outside $H_{i}$ by zero and obtain thus a natural embedding of $\mathscr{H} \mathscr{S}\left(H_{i}, H_{j}\right)$ into $\mathscr{H} \mathscr{S}(H)$. The space $\mathscr{B}_{\text {res }}(H)$ is a subalgebra of $\mathscr{B}(H)$ since the collection of Hilbert-Schmidt operators is a 2 -sided ideal in $\mathscr{B}(H)$. Hence it is also a Lie subalgebra of the Lie algebra $\mathscr{B}(H)$. The algebra $\mathscr{B}_{\text {res }}(H)$ becomes a Banach algebra if we equip it with the norm $\|\cdot\|_{2}$ defined by

$$
\|u\|_{2}=\|u\|+\sum_{i \neq j}\left\|u_{i j}\right\|_{\mathscr{H} \mathscr{S}} .
$$

Since the adjoint of a Hilbert-Schmidt operator is again Hilbert-Schmidt, it is clear that $\mathscr{B}_{\text {res }}(H)$ is stable under "taking adjoints". If $G L(H)$ denotes the group of invertible elements in $\mathscr{B}(H)$, then we consider

Definition 2.1.12. The restricted linear group, $G L_{\text {res }}(H)$, consists of $\left\{g \mid g \in G L(H) \cap \mathscr{B}_{\text {res }}(H)\right\}$.

To see that $G L_{\text {res }}(H)$ is indeed a group, one merely has to show that if $g=\left(g_{i j}\right)$ belongs to $G L_{\mathrm{res}}(H)$ then its inverse $g^{-1}=\left(\left(g^{-1}\right)_{i j}\right)$ also belongs to $G L_{\text {res }}(H)$. Now, the relation

$$
g_{i i}\left(g^{-1}\right)_{i i}=\operatorname{Id}_{H_{1}}-\sum_{j \neq i} g_{i j}\left(g^{-1}\right)_{j i},
$$

shows first of all that for all $i, 1 \leq i \leq m$, both $g_{i i}$ and $\left(g^{-1}\right)_{i i}$ are Fredholm operators, i.e. they have a finite dimensional kernel and cokernel. Next one considers the relation

$$
g_{21}\left(g^{-1}\right)_{11}+g_{22}\left(g^{-1}\right)_{21}+\sum_{j>2} g_{2 j}\left(g^{-1}\right)_{j 1}=0 .
$$

Since the operator $g_{21}$ is Hilbert-Schmidt and the operators $\left(g^{-1}\right)_{11}$ and $g_{22}$ are Fredholm, the operator $\left(g^{-1}\right)_{21}$ has to be Hilbert-Schmidt too. Continuing in this fashion, one shows that all $\left(g^{-1}\right)_{i j}$ with $i \neq j$ are Hilbert-Schmidt. In other words, $G L_{\text {res }}(H)$ consists of the invertible elements of $B_{\text {res }}(H)$. As such, it is in a natural way a Banach Lie group with Lie algebra $B_{\text {res }}(H)$.

The analogue of the unitary group $U(H)$ in this context is: 
Definition 2.1.13. The restricted unitary group, $U_{\text {res }}(H)=G L_{\mathrm{res}}(H) \cap U(H)$.

Both $U_{\text {res }}(H)$ and $G L_{\text {res }}(H)$ are natural generalizations of the restricted unitary and general linear group, introduced in chapter 6 of [23]. The Lie algebra of $U_{\text {res }}(H)$ consists of

$$
\mathfrak{u}_{\text {res }}(H)=\left\{X \mid X \in \mathfrak{B}_{\text {res }}(H), X^{*}=-X\right\}
$$

This is a real Lie subalgebra of $\mathfrak{B}_{\text {res }}(H)$ and the Lie algebra $\mathfrak{B}_{\text {res }}(H)$ can be written as

$$
\mathfrak{B}_{\text {res }}(H)=\mathfrak{u}_{\text {res }}(H) \oplus i . \mathfrak{u}_{\text {res }}(H) .
$$

In other words $\mathfrak{B}_{\text {res }}(H)$ is the complexification of $\mathfrak{u}_{\text {res }}(H)$. On the group level this corresponds to the fact that the group $G L_{\mathrm{res}}(H)$ possesses a "polar decomposition" of which $U_{\text {res }}(H)$ forms the unitary component. For, consider the sets

$$
\begin{aligned}
P(H) & =\left\{A \mid A \in G L(H), A=A^{*} \text { and } A>0\right\} \text { and } \\
P_{\text {res }}(H) & =\mathfrak{B}_{\text {res }}(H) \cap P(H) .
\end{aligned}
$$

On $P_{\text {res }}(H)$ we put the topology induced by $\mathfrak{B}_{\text {res }}(H)$. Since the map $A \mapsto \sqrt{A}$ from $P_{\text {res }}(H)$ to $P(H)$ is locally given by a convergent power series in $A$, this map is in fact a continuous map from $P_{\text {res }}(H)$ to itself. Thus we get

Proposition 2.1.14. The map $(u, p) \mapsto u p$ from $U_{\text {res }}(H) \times P_{\text {res }}(H)$ to $G L_{\text {res }}(H)$ is a homeomorphism.

Proof. The inverse of this map is

$$
g \longmapsto\left(g\left(\sqrt{g^{*} g}\right)^{-1}, \sqrt{g^{*} g}\right)
$$

and we have just seen that it is continuous.

With each $g$ in $G L_{\text {res }}(H)$ we can associate the flag

$$
0 \subset g H_{1} \subset g\left(H_{1} \oplus H_{2}\right) \subset \cdots g\left(H_{1} \oplus \cdots \oplus H_{i}\right) \subset \cdots \subset H .
$$

From the definition of $G L_{\text {res }}(H)$ one sees directly that this flag belongs to $\mathfrak{F}$.

The group $U_{\text {res }}(H)$ acts already transitively on $\mathfrak{F}$. Let $F=\left\{F_{1}, \ldots, F_{m}\right\}$ belong to $₹$. From the definition of $₹$ we know that there is for each $i$, $1 \leq i \leq m$, an isometry $u_{i}$ between $H_{i}$ and $F_{i}$. If we put $u=u_{1} \oplus \cdots \oplus u_{m}$, then the condition defining $\mathfrak{F}$ implies that $u$ belongs to the group $U_{\text {res }}(H)$ and that $F=u\left(F^{(0)}\right)$.

The stabilizer in $G L_{\text {res }}(H)$ of the basic flag is the "parabolic subgroup" 
$P=\left\{g \mid g \in G L_{\mathrm{res}}(H), g=\left(\begin{array}{cccc}g_{11} & \cdots & \cdots & g_{1 m} \\ 0 & \ddots & & \vdots \\ \vdots & \ddots & \ddots & \vdots \\ 0 & \cdots & 0 & g_{m m}\end{array}\right)\right.$, with $\left.g_{i i} \in G L_{\mathrm{res}}\left(H_{i}\right), 1 \leq i \leq m\right\}$

Thus we can identify $\mathbb{F}$ also with the homogeneous space $G L_{\text {res }}(H) / P$. Let $\tau: G L_{\text {res }}(H) \rightarrow \mathscr{F}$ be the projection $\tau(g)=g \cdot F^{(0)}$. On $\mathfrak{F}$ we will put a Hilbert manifold structure that makes $\tau$ into an open submersion. This will be discussed in the next subsection.

Remark 2.1.15. It will be clear that for the spaces $\mathfrak{F}(\mathscr{N}), \mathfrak{F}(\mathscr{C})$ and $\mathfrak{F}(\mathscr{B})$ the corresponding general linear group consists of those $g=\left(g_{i j}\right)$ in $G L(H)$ such that respectively

$$
\begin{aligned}
g_{i j} \in \mathscr{N}\left(H_{j}, H_{i}\right) & \text { for all } i \neq j, \\
g_{i j} \in \mathscr{C}\left(H_{j}, H_{i}\right) & \text { for all } i \neq j, \\
g_{i j} \in \mathscr{H} \mathscr{S}\left(H_{j}, H_{i}\right) & \text { for all } i<j .
\end{aligned}
$$

2.2. The manifold structure of $\mathfrak{F}$. In this subsection we discuss the Hilbert manifold structure on $\mathfrak{F}$ and some decompositions of open subsets in $G L_{\text {res }}(H)$. From the definition of the parabolic group $P$ one sees directly that the Lie algebra of $P$ is given by

$$
L(P)=\left\{g \mid g=\left(g_{i j}\right) \in B_{\text {res }}(H), g_{i j}=0 \text { for all } i>j\right\}
$$

and that a complement of $L(P)$ in $B_{\text {res }}(H)$ is the Hilbert space $\left(E,\|\cdot\|_{\mathscr{H} \mathscr{S}}\right)$ with

$$
E=\bigoplus_{\substack{1 \leq j \leq m-1 \\ i>j}} \mathscr{H} \mathscr{S}\left(H_{j}, H_{i}\right) .
$$

From section 6.1 in [3], we know then that the homogeneous space $\mathfrak{F}=G L_{\text {res }}(H) / P$ carries an analytic $E$-manifold structure for which $\tau$ is a submersion and for which the natural action of $G L_{\text {res }}(H)$ on $\mathfrak{F}$ is analytic.

Next we give descriptions of some open subsets in $G L_{\text {res }}(H)$ that will be needed later on. Consider for each $k, 1 \leq k \leq m-1$, the set $\Omega(k)$ in $G L_{\text {res }}(H)$ given by

$\Omega(k)=\left\{g \in G L_{\mathrm{res}}(H) \mid\left(\begin{array}{ccc}g_{11} & \cdots & g_{1 i} \\ \vdots & & \vdots \\ g_{i 1} & \cdots & g_{i i}\end{array}\right) \in G L_{\mathrm{res}}\left(H_{1} \oplus \cdots \oplus H_{i}\right)\right.$ for all $\left.i \leq k\right\}$.

Since we have for each $i, 1 \leq i \leq m$, a continuous surjection from $\mathscr{B}_{\text {res }}(H)$ onto $\mathscr{B}_{\text {res }}\left(H_{1} \oplus \cdots \oplus H_{i}\right)$ given by 


$$
b \longmapsto\left(\begin{array}{ccc}
b_{11} & \cdots & b_{1 \imath} \\
\vdots & & \vdots \\
b_{i 1} & \cdots & b_{i i}
\end{array}\right),
$$

the set $\Omega(k)$ is open and, as in the finite dimensional case, it can be decomposed. For, let $U_{-}(k)$ and $P(k)$ be the Lie subgroups of $G L_{\text {res }}(H)$ defined by

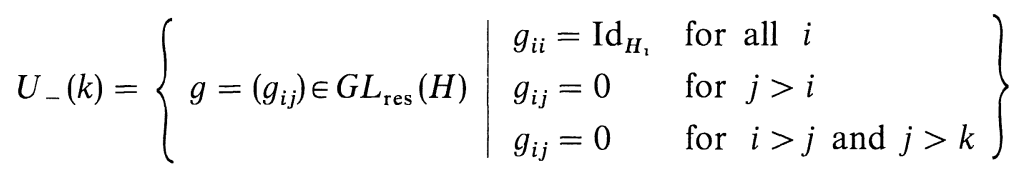

and

$$
P(k)=\left\{g=\left(g_{i j}\right) \in G L_{\text {res }}(H) \mid g_{i j}=0 \text { if } i>j \text { and } j \leq k\right\} .
$$

Clearly $P(k) \cap U_{-}(k)=\left\{\operatorname{Id}_{H}\right\}$ and this gives you the uniqueness in

Lemma 2.2.1. The map $(u, p) \mapsto u p$ from $U_{-}(k) \times P(k) \rightarrow G L_{\text {res }}(H)$ determines a homeomorphism between $U_{-}(k) \times P(k)$ and $\Omega(k)$.

Proof. We use induction on $k$ to show the result. Let $g$ be an element in $\Omega(1)$. Then we know that $g_{11}$ is invertible and if we define $u(1) \in U_{-}(1)$ by $u(1)_{r 1}=-g_{r 1} g_{11}^{-1}$ for all $r \geq 2$, then one sees directly that $u(1) g$ belong to $P(1)$. Assume now that we know $\Omega(l)=U_{-}(l) P(l)$. Since we have $\Omega(l) \supset$ $\Omega(l+1)$ and $U_{-}(l)<U_{-}(l+1)$, we may assume that $g \in \Omega(l+1)$ belongs to $P(l)$. Hence the condition $g \in \Omega(l+1)$ means that $g_{l+1, l+1}$ is invertible. Define $u(l+1)=\left(u_{i j}\right)$ in $U_{-}(l+1)$ by $u_{j l+1}=-g_{j l+1}\left(g_{l+1, l+1}\right)^{-1}$ for $j>l+1$ and $u_{i j}=0$ if $i>j$ and $j \neq l+1$. Then $u(l+1) \cdot g$ belongs to the parabolic group $P(l+1)$. This proves the lemma.

As in the finite dimensional case we call $\Omega(m-1)=U_{-}(m-1) \cdot P$ the big cell of $\mathfrak{F}$ and we also write $\Omega$ and $U_{-}$instead of $\Omega(m-1)$ and $U_{-}(m-1)$.

From this lemma we see that the restriction of $\tau$ to $U_{-}$gives you a diffeomorphism $u \mapsto u F^{(0)}$ between $U_{-}$and the open neighborhood $\tau(\Omega)$ of $F^{(0)}$. Clearly the group $U_{-}$is diffeomorphic to the Hilbert space $E$. Note that from the definition of $\Omega$ one can conclude directly that

$$
\tau(\Omega)=\left\{F=\left(F_{i}\right) \in \mathfrak{F} \mid \underset{j \leq l}{\bigoplus} p_{j}: \underset{j \leq l}{\bigoplus} F_{j} \longrightarrow \underset{j \leq l}{\bigoplus} H_{j} \text { is a bijection for all } l \leq m\right\} .
$$

This characterization of $\tau(\Omega)$ tells you how to choose around a general point of $\mathfrak{F}$ a concrete neighborhood diffeomorphic to $E$. This requires, however, the introduction of the following notation.

Notation 2.2.2. If $W$ is closed subspace of $H$, then we denote the 
orthogonal projection on $W$ by $p_{W}$.

Consider a $F=\left(F_{1}, \ldots, F_{m}\right)$ in $\mathfrak{F}$. Then the analogue of $\tau(\Omega)$ for $F$ is

$$
U_{F}=\left\{V=\left(V_{i}\right) \text { in } \mathfrak{F} \mid \bigoplus_{i \leq l} p_{F_{l}}: \underset{i \leq l}{\oplus} V_{i} \longrightarrow \underset{i \leq l}{\bigoplus} F_{i} \text { is a bijection for all } 1 \leq l \leq m\right\} \text {. }
$$

Since $\mathfrak{F}=U_{\text {res }}(H) \cdot F^{(0)}$, we have for all $F$ and $G$ in $\mathfrak{F}$ that, if $i \neq j$, the map $p_{F_{i}}: G_{j} \rightarrow F_{i}$ is a Hilbert-Schmidt operator. Hence, if $V$ belongs to $U_{F}$, then there is a unique operator $A$ in $\bigoplus_{1 \leq j \leq m} \mathscr{H} \mathscr{S}\left(F_{j}, F_{i}\right)$ such that for all $i$, $1 \leq i \leq m$,

$$
V(i)=\{x+A(x) \mid x \in F(i)\} .
$$

This is why we call $V$ also the graph of $A$ and we write $V=\operatorname{graph}(A)$.

It is convenient to have a special name for the flags in $U_{F}$.

Definition 2.2.3. A flag $V$ in $U_{F}$ is called transversal to $F$.

Let $g_{F}$ be an element of $U_{\text {res }}(H)$ such that $g_{F} \cdot F^{(0)}=F$. Instead of the big cell $\Omega$ in $G L_{\text {res }}(H)$ with respect to the decomposition $H=H_{1} \oplus \cdots \oplus H_{m}$, we could also have introduced a big cell with respect to $H=F_{1} \oplus \cdots \oplus F_{m}$ and it will be clear that this set can be written as

$$
g_{F} U_{-} P\left(g_{F}^{-1}\right) .
$$

Consequently, we get for $U_{F}$ that

$$
U_{F}=\left\{g_{F} u p\left(g_{F}\right)^{-1} F \mid \text { with } u \in U_{-} \text {and } p \in P\right\}=\tau\left(g_{F} U_{-} P\right) .
$$

Then we can define for each $F$ in $\mathfrak{F}$ a diffeomorphism $\varphi_{F}: U_{F} \rightarrow E$ by

$$
\varphi_{F}\left(g_{F} u F^{(0)}\right)=u-\mathrm{Id}
$$

Each $\left(U_{F}, \varphi_{F}\right)$ is a concrete chart around $F$ for the $E$-manifold structure on $\mathfrak{F}$.

We have obtained now a concrete description of the manifold structure on $\mathfrak{F}$ :

Proposition 2.2.4. The $\left(U_{F}, \varphi_{F}\right)$ are the charts of the analytic E-manifold structure on $\mathfrak{F}$.

Proof. It is sufficient to show for each $U_{F^{(1)}}$ and $U_{F^{(2)}}$ with $U_{F^{(1)}} \cap U_{F^{(2)}} \neq \varnothing$ that

$$
\varphi_{F^{(2)}} \circ \varphi_{F^{(1)}}^{-1}: \varphi_{F^{(1)}}\left(U_{F^{(1)}} \cap U_{F^{(2)}}\right) \longrightarrow \varphi_{F^{(2)}}\left(U_{F^{(1)}} \cap U_{F^{(2)}}\right)
$$

is an analytic map. From the step by step decomposition described in Lemma 2.2.1 follows that the $U_{-}$-component of $\left(g_{F^{(2)}}\right)^{-1} g_{F^{(1)}} u$ actually depends analytically on $u$. This proves the proposition. 
2.3. The connected components of $G L_{\text {res }}(\boldsymbol{H})$. Let $g=\left(g_{i j}\right)$ be an element of $G L_{\text {res }}(H)$. Recall that in the proof that $G L_{\text {res }}(H)$ consists of the invertible elements in $\mathscr{B}_{\text {res }}(H)$, we have shown that each $g_{i i}$ is a Fredholm operator. The collection of Fredholm operators on a Hilbert space $K$ is an open part of the space $\mathscr{B}(K)$. Its connected components are given by the index, which is defined as

$$
\text { ind }(B)=\operatorname{dim}(\operatorname{ker}(B))-\operatorname{dim}(\operatorname{coker}(B))
$$

where $B$ is a Fredholm operator on $K$. Since all off-diagonal operators are Hilbert-Schmidt and hence compact, the operator

$$
\tilde{g}=\left(\begin{array}{ccc}
g_{11} & & 0 \\
& \ddots & \\
0 & & g_{m m}
\end{array}\right) \text {, where } g=\left(g_{i j}\right) \in G L_{\mathrm{res}}(H),
$$

is a Fredholm operator of index zero. Hence we have that the indices of the $\left\{g_{i i} \mid 1 \leq i \leq m\right\}$ satisfy

$$
\sum_{i=1}^{m} \text { ind }\left(g_{i i}\right)=0 \text { and ind }\left(g_{k k}\right)=0 \text { if } m_{k}<\infty .
$$

These relations lead to the introduction of the subgroup $Z$ of $\mathbf{Z}^{m}$ defined by

$$
Z=\left\{z=\left(z_{i}\right) \in \mathbf{Z}^{m} \mid \sum_{i=1}^{m} z_{i}=0, z_{k}=0 \text { if } m_{k}<\infty\right\}
$$

The standard properties of the index imply that the map $i: G L_{\mathrm{res}}(H) \rightarrow Z$,

$$
\left.g \longmapsto \text { (ind }\left(g_{11}\right), \ldots, \text { ind }\left(g_{m m}\right)\right),
$$

is a continuous group homomorphism. Hence the sets

$$
G L_{\mathrm{res}}^{(z)}(H)=\left\{g: g \in G L_{\mathrm{res}}(H), i(g)=z\right\}, \text { with } z \in Z,
$$

are open. In fact, they are exactly the connected components of $G L_{\mathrm{res}}(H)$, for

Proposition 2.3.1. For each $z \in Z$, the set $G L_{\mathrm{res}}^{(z)}(H)$ is non-empty and connected.

Proof. Let $z=\left(z_{i}\right)$ be in $Z$ and let $h_{i} \in \Phi\left(H_{i}\right)$ be such that ind $\left(h_{i}\right)=z_{i}$. Then

$$
h=\left(\begin{array}{lll}
h_{1} & & 0 \\
& \ddots & \\
0 & & h_{m}
\end{array}\right)
$$


belongs to $\Phi(H)$ and has index zero. Therefore one can add to $h$ an isomorphism between the kernel of $h$ and the orthogonal complement of the image of $h$ to obtain an element of $G L_{\text {res }}^{(z)}(H)$. This shows that $i$ is surjective. As for the connectedness it suffices to show that $G L_{\text {res }}^{(0)}(H)$ is connected. First one notes that, since $P$ is homeomorphic to

$$
\prod_{i=1}^{m} G L\left(H_{i}\right) \times \prod_{j<i} \mathscr{H} \mathscr{S}\left(H_{j}, H_{i}\right)
$$

and all the $G L\left(H_{i}\right)$ are connected, (see [20]), the group $P$ is connected. Next we show that each element of $G L_{\text {res }}^{(0)}(H)$ can be joined by a continuous path to an element of $P$. For an element $g=u p$ in $\Omega=U_{-} P$ it is clear how to proceed: the map $t \mapsto\{\mathrm{Id}+(1-t)(u-\mathrm{Id})\} p$ joins $g$ with $p$. A general element $g$ is first joined with an element in $\Omega(1)$. For, if $g_{11}$ is not invertible, then there is a bijection $E$ between $\operatorname{ker}\left(g_{11}\right)$ and $\mathfrak{I}\left(g_{11}\right)^{\perp} \cap H_{1}$ and we extend $E$ by zero on $\operatorname{ker}\left(g_{11}\right)^{\perp}$ to get an element $E$ of $\mathscr{B}_{\text {res }}(H)$. It is no restriction to assume $\|E\|_{2}<\|g\|_{2}$. Then we know that $g+t E$ belongs to $G L_{\mathrm{res}}^{(0)}(H)$ for all $t \in[0,1]$ and by construction $g+E$ belongs to $\Omega(1)$ and we can write $g+E=u_{1} p_{1}$. The map $t \mapsto\left\{\mathrm{Id}+(1-t)\left(u_{1}-\mathrm{Id}\right)\right\} p_{1}$ joins $g+E$ with $p_{1}$. By adding a small finite dimensional operator in $\mathscr{B}\left(H_{2}\right)$, one reduces the case to an element in $\Omega(2)$ that can be linked in the same way to an element of $P(2)$. Continuing in this fashion one finds a continuous path from $g$ to an element of $P$. This proves the assertion.

This Proposition is the extension to flag varieties of Proposition 6.2.4 in [23].

Since the parabolic group $P$ is connected, we see that

Corollary 2.3.2. The connected components of $\mathfrak{F}$ are given by

$$
\mathfrak{F}^{(z)}=\left\{g \cdot F^{(0)} \mid g \in G L_{\text {res }}^{(z)}(H)\right\} .
$$

Remark 2.3.3. A holomorphic line bundle $L$ over $\mathfrak{F}$ consists simply of a collection of holomorphic line bundles $\left\{L_{z} \rightarrow \mathfrak{F}^{(z)} \mid z \in Z\right\}$. Therefore we restrict our attention to holomorphic line bundles over $\mathfrak{F}^{(0)}$ in the third section.

2.4. A special covering of $\mathfrak{F}$. In this subsection we choose a suitable orthonormal basis of $H$ and we introduce a collection of charts of $\mathfrak{F}$ that can be described completely in terms of the index set of this orthonormal basis. In particular these charts cover $\mathfrak{F}$ and enable you to give a combinatorial description of the Birkhoff decomposition of $G L_{\text {res }}(H)$ and to construct concretely a collection of holomorphic line bundles over $\mathfrak{F}^{(0)}$.

Let $\left\{e_{s} \mid s \in S_{i}\right\}, 1 \leq i \leq m$, be an orthonormal basis of $H_{i}$. Recall that $\operatorname{dim}\left(H_{i}\right)=m_{i}$ for all $i, 1 \leq i \leq m$. Hence we can write

$$
S_{i}=\left\{s_{i}(k) \mid 1 \leq k<m_{i}+1\right\}
$$


On $S_{i}$ we define a total order by

$$
s_{i}(k) \geq s_{i}(l) \Longleftrightarrow k \geq l \text {. }
$$

These orders we compose to a total order on the index set $S=\bigcup_{i=1}^{m} S_{i}$ by requiring that

$$
s_{j}<s_{i} \text { for all } s_{i} \in S_{i} \text { and all } s_{j} \in S_{j} \text { with } j>i \text {. }
$$

Now that we have an orthonormal basis $\left\{e_{s} \mid s \in S\right\}$ of $H$ with a totally ordered index set $S$, we can associate to each bounded $g$ in $\mathscr{B}(H)$ an $S \times S$-matrix $[g]=\left(g_{s t}\right)$ with matrix coefficients

$$
g_{\mathrm{st}}=\left\langle g\left(e_{t}\right), e_{\mathrm{s}}\right\rangle, \text { where } s \text { and } t \text { are in } S .
$$

Notation 2.4.1. Let $\mathfrak{g l}(S)$ be the collection of $S \times S$-matrices corresponding to operators in $\mathscr{B}_{\text {res }}(H)$.

The context has been chosen such that the product of two elements in $\mathrm{gl}(S)$ is again in $\mathrm{gl}(S)$ and therefore $\mathrm{gl}(S)$ is a Lie algebra. In $\mathrm{gl}(S)$ we have the Lie subalgebra $\mathfrak{g l}(\infty)$ corresponding to the matrices of the operators

Definition 2.4.2. An operator $g$ in $\mathscr{B}(H)$ is called a "finite-size" operator if it has only a finite number of non-zero matrix coefficients w.r.t. the $\left\{e_{s} \mid s \in S\right\}$.

Remark 2.4.3. If $m=2$ and $m_{1}=m_{2}=\infty$, then $S=\mathbf{Z}$. In [18] it was shown that the Lie algebra $A_{\infty}$ can be realized as a central extension of the collection $\overline{\mathfrak{g l}(\infty)}$ of $\mathbf{Z} \times \mathbf{Z}$-matrices $g=\left(g_{i j}\right)$ of "finite-width", i.e. satisfying $g_{i j}=0$ if $|i-j|>N$ for some $N$. The composition of such matrices is always defined and from this point of view $\mathrm{gl}(S)$ can be seen as a complete bounded version of $\overline{\mathfrak{g l}(\infty)}$. The central extension defining $A_{\infty}$ also occurs naturally in our geometric framework, see subsection 4 of the next section.

In the sequel we will frequently use some notations related to subsets of $S$.

Notation 2.4.4. The number of elements in a subset $A$ of $S$ is denoted by $\#$ A.

Notation 2.4.5. If $A$ is a non-empty subset of $S$, then we denote the closure of the span of the $\left\{e_{s} \mid s \in A\right\}$ by $H_{A}$. If $A$ is empty, then $H_{A}$ denotes the space $\{0\}$. It is convenient to denote the orthogonal projection onto $H_{A}$ by $p_{A}$.

Maps between subsets of $S$ have a direct translation to partial isometries between closed subspaces of $H$, i.e.

Notation 2.4.6. If $A$ and $B$ are subsets of $S$ and $\tau: A \rightarrow B$ is a map with uniformly bounded finite fibers, then we denote by $\underline{\tau}$ the mapping from $H_{A}$ to $H_{B}$ given by 


$$
\underline{\tau}\left(\sum_{t \in A} \lambda_{t} e_{t}\right)=\sum_{t \in A} \lambda_{t} e_{\tau(t)}
$$

Now that we have chosen the orthonormal basis $\left\{e_{s} \mid s \in S\right\}$, we can introduce "diagonal operators" in $\mathscr{B}_{\text {res }}(H)$. Suppose that we have a set of bounded complex numbers

$$
\left\{\delta_{s} \mid s \in S, \delta_{s} \in \mathbf{C} \text { and }\left|\delta_{s}\right| \leq M \text { for all } s \in S\right\} .
$$

Then we can associate to it a diagonal operator $\operatorname{diag}\left(\delta_{s}\right)$ in $\mathscr{B}(H)$ by

$$
\operatorname{diag}\left(\delta_{s}\right)\left(\sum_{t \in S} \lambda_{t} e_{t}\right)=\sum_{t \in S} \delta_{t} \lambda_{t} e_{t}
$$

Inside $G L_{\text {res }}(H)$ we have then the "maximal torus"

$$
T=\left\{g \mid g \in G L_{\text {res }}(H), g=\operatorname{diag}\left(\delta_{s}\right)\right\} .
$$

Clearly $T$ is commutative and it is a straightforward verification to show that the centralizer of $T$ inside $G L(H)$ is equal to $T$. Hence we have

Lemma 2.4.7. The centralizer $Z(T)$ of $T$ in $G L_{\mathrm{res}}(H)$ is equal to $T$.

Each permutation $\sigma$ of $S$ determines a unitary map $\underline{\sigma}: H \rightarrow H$ as in notation 2.4.6. With the help of the matrix, one shows that the normalizer of $T$ in $G L(H)$ consists of

$$
\{t \cdot \underline{\sigma} \mid t \in T, \sigma \text { a permutation of } S\} \text {. }
$$

Hence, if we define the subgroup $W$ of $U_{\text {res }}(H)$ as

$$
W=\left\{\underline{\sigma} \mid \underline{\sigma} \in U_{\text {res }}(H), \sigma \text { a permutation of } S\right\},
$$

then we have

Corollary 2.4.8. The normalizer $N(T)$ of $T$ in $G L_{\text {res }}(H)$ is the semi-direct product of $W$ and $T$. In particular, we see that $W$ is isomorphic to $N(T) / Z(T)$ and we call $W$ the Weyl group of $T$.

To each $\underline{\sigma}$ in $W$, corresponds a partition $\Sigma=\bigcup_{i \geq 1} \Sigma_{i}$ of $S$, where $\Sigma_{i}=\sigma\left(S_{i}\right)$. The concrete description of which partitions occur in this way, brings one in a natural way to the consideration of subsets of $S$ that are "equal up to a finite set". Therefore we define

Definition 2.4.9. If $A$ and $B$ are subsets of $S$, then we call $A$ and $B$ commensurable (notation $A \approx B$ ) if $A-\{A \cap B\}$ and $B-\{A \cap B\}$ are finite. We write $i(A, B)$ for the number 


$$
\#\{A-\{A \cap B\}\}-\#\{B-\{A \cap B\}\} .
$$

Thus commensurability is equivalent to: the orthogonal projection $p_{H_{B}}$ : $H_{A} \rightarrow H_{B}$ is a Fredholm operator with index $i(A, B)$. Let $\Sigma=\left\{\Sigma_{i} \mid 1 \leq i \leq m\right\}$ be an arbitrary partition of $S$ into $m$ disjoint parts. Then this partition corresponds to an element of $W$, if and only if the following two conditions hold:

$$
\begin{array}{cc}
\# \Sigma_{i}=\# S_{i} & \text { for all } i, 1 \leq i \leq m, \text { and } \\
\Sigma_{i} \approx S_{i} & \text { for all } i, 1 \leq i \leq m
\end{array}
$$

To any partition $\Sigma$ satisfying these conditions there corresponds a flag $F_{\Sigma}$ in If given by

$$
0 \subset H_{\Sigma_{1}} \subset H_{\Sigma_{1} \cup \Sigma_{2}} \subset \cdots \subset H_{S}=H .
$$

For simplicity we denote for each $\Sigma$ satisfying (5) and (6) the open set $U_{F_{\Sigma}}$ in $\mathfrak{F}$ also by $U_{\Sigma}$.

In the sequel we will make use of the following notion

Definition 2.4.10. An element in $H$ is said to be of order $s, s \in S$, if it has the form

$$
h=a_{s} e_{s}+\sum_{\substack{t \in S \\ t<s}} a_{t} e_{t}, \text { with } a_{s} \neq 0,
$$

Notation 2.4.11. If $W$ is a subspace of $H$ then the union of all the elements in $W$ of some order $s$ in $S$ and $\{0\}$ is called the space of elements of finite order in $W$ and is denoted by $W_{\text {fin }}$.

For each $z$ in $Z$, we denote the collection of partitions $\Sigma$ of the index set $S$ such that $F_{\Sigma}$ belongs to $\mathscr{F}^{(z)}$, by $\mathscr{S}(z)$. The basic property of the $\left\{F_{\Sigma} \mid \Sigma \in \mathscr{S}(z)\right\}$ is

Proposition 2.4.12. For each flag $F=(F(1), \ldots, F(m))$ in $\mathfrak{F}^{(z)}$ there is a $\Sigma$ in $\mathscr{S}(z)$ such that $F$ is transversal to $F_{\Sigma}$.

Proof. Let $g \in G L_{\text {res }}(H)$ be such that $F=g \cdot F^{(0)}$. First we show that there is a $\Sigma_{1}$ commensurable with $S_{1}$ and with $\# S_{1}=\# \Sigma_{1}$, such that $\left.p_{H_{1}} \circ g\right|_{H_{1}}$ is an isomorphism between $H_{1}$ and $H_{\Sigma_{1}}$. Since $p_{1}\left(g\left(H_{1}\right)\right)$ has finite codimension in $H_{1}$, we can find a $S_{1}(n)=\left\{s_{1}(k), k \geq n\right\}, n \geq 0$, such that $F(1)=g\left(H_{1}\right)$ projects surjectively onto $H_{S_{1}(n)}$. The kernel of this projection has a basis $\left\{h_{j} \mid 1 \leq j \leq N\right\}$ of elements of finite order, i.e.

$$
h_{j}=e_{s_{j}}+\sum_{\substack{t \in S \\ t<s_{j}}} \alpha_{j}(t) e_{t}, \text { where } s_{i} \neq s_{j} \text { for } i \neq j .
$$

It is clear that we can take $\Sigma_{1}=S_{1}(n) \cup\left\{s_{j} \mid 1 \leq j \leq N\right\}$. The other parts of 
the desired partition $\Sigma$ of $S$ are constructed step by step from $\Sigma_{1}$. For, assume that we have found disjoint $\left\{\Sigma_{j} \mid j \leq i\right\}$ with $\Sigma_{j} \approx S_{j}$ and $\# S_{j}=\# \Sigma_{j}$ such that the orthogonal projection of $F(j)$ onto $\bigotimes_{\ell \leq j} H_{\Sigma_{\ell}}$ is a bijection for all $j \leq i$. Then we know that $p_{i+1}\left(g\left(H_{i+1}\right)\right)$ has finite codimension in $H_{i+1}$. So there exists a subset $\tilde{S}_{i+1}$ of $S_{i+1}$, commensurable with $S_{i+1}$ and disjoint of $\Sigma_{1} \cup \cdots \Sigma_{i}$, such that $F(i+1)$ projects surjectively onto $H_{\Sigma_{1}} \oplus \cdots \oplus H_{\Sigma_{i}} \oplus H_{\tilde{S}_{1+1}}$. The kernel of this projection is again finite dimensional and has a basis of elements of different order. As $\Sigma_{i+1}$ one takes then the union of $\tilde{S}_{i+1}$ and the orders of the elements in this basis. In this way we obtain after a finite number of steps the desired partition $\Sigma$ of $S$.

This proposition is a generalization of Proposition 7.16 in [23].

Remark 2.4.13. Since $F_{\Sigma}$ is transversal to $F_{\Pi}$ if and only if $\Sigma=\Pi$, we can conclude from this proposition directly that $\mathfrak{F}^{(0)}$ is no longer compact if $H$ is infinite dimensional.

For each $\Sigma$ in $\mathscr{S}=\bigcup_{z \in Z} \mathscr{S}(z)$, the elements of finite order in $F_{\Sigma}(j)$ span a dense subspace of $F_{\Sigma}(j)$ for all $j, 1 \leq j \leq m$. By combining this with Proposition 2.4.12 we get the following generalization of proposition 7.3.2 in [23].

Corollary 2.4.14. For each flag $F$ in $\mathfrak{F}$ and for each $j, 1 \leq j \leq m$, the space $F(j)_{\text {fin }}$ forms a dense subspace of $F(j)$.

For each flag $F=(F(0), \ldots, F(m))$ in $\mathfrak{F}^{(z)}$ we can concretely describe a $\Sigma(F)$ in $\mathscr{S}(z)$ such that $F$ is transversal to $F_{\Sigma(F)}$. Namely for each $1 \leq i \leq m$ we put

$$
\Sigma(F)(i)=\{s \mid s \in S, F(i) \text { contains an element of order } s\}
$$

and

$$
\Sigma(F)_{i}=\Sigma(F)(i)-\Sigma(F)(i-1) \text { for } i>1 .
$$

Clearly each $F(i)$ projects bijectively onto $H_{\Sigma(F)(i)}$ and therefore $\Sigma(F)=\left\{\Sigma(F)_{i}\right\}$ belongs to $\mathscr{S}(z)$. Next we consider flags that give the same partition in this way.

If $\Sigma \in \mathscr{S}(z)$, then we write

$$
\mathfrak{F}_{\Sigma}=\{F \mid F \in \mathfrak{F}, \Sigma(F)=\Sigma\}
$$

Let $U_{0}$ be the subgroup of $G L_{\text {res }}(H)$ of all operators with a unipotent lower triangular matrix, i.e.

Then we want to show

$$
U_{0}=\left\{u \mid u \in G L_{\mathrm{res}}(H) \text {, for all } s \in S, u\left(e_{s}\right)=e_{s}+\sum_{t<s} u_{t s} e_{t}\right\} .
$$


Proposition 2.4.15. The subset $\mathfrak{F}_{\Sigma}$ is exactly the $U_{0}$-orbit through $F_{\Sigma}$.

Proof. From the form of an operator $u$ in $U_{0}$, one sees directly that for each element $h$ of order $s$, the element $u(h)$ has also order $s$. Thus we have that $\Sigma(u F)=\Sigma(F)$ for each $F$ in $\Sigma$.

Assume now that $F$ belongs to $\mathfrak{F}_{\Sigma}$. Since $F(i)$ projects bijectively onto $F_{\Sigma}(i)$ for all $i, 1 \leq i \leq m$, the flag $F$ is the graph of an operator $T$ in

$$
\bigoplus_{j=1}^{m-1} \bigoplus_{j<i} \mathscr{H} \mathscr{S}\left(H_{\Sigma_{j}}, H_{\Sigma_{l}}\right) \text {. }
$$

In particular this means that there is an $u$ in $G L_{\text {res }}(H)$ such that $u\left(F_{\Sigma}\right)=F$ and for all $s_{i} \in \Sigma_{i}, 1 \leq i \leq m$,

$$
u\left(e_{s_{l}}\right)=e_{s_{l}}+\sum_{j>i} \sum_{s_{J} \in \Sigma_{J}} T_{s_{J} s_{l}} e_{s_{J}} \text {, if } i<m \text { and } u\left(e_{s_{l}}\right)=e_{s_{l}} \text { if } i=m .
$$

The fact that $F$ belongs to $\mathfrak{F}_{\Sigma}$ can be expressed completely in term of the coefficients $\left\{T_{s_{j} s_{l}} \mid s_{i} \in \Sigma_{i}, s_{j} \in \Sigma_{j}, j>i\right\}$. Namely, it is equivalent to

$$
T_{s_{s} s_{i}}=0, \text { if } s_{j}>s_{i} \text {. }
$$

If namely $T_{s_{j} s_{t}} \neq 0$ for some $s_{j}$ with $s_{j}>s_{i}$, then the element $u\left(e_{s_{t}}\right)$ will be of some order $s \notin \Sigma(i)$ and hence $\Sigma(F)(i) \neq \Sigma(i)$. This contradicts the fact that $F$ belongs to $\mathfrak{F}_{\Sigma}$. By definition, the operator $u$ defined by (7) belongs to $U_{0}$, if condition (8) is satisfied. This concludes the proof of the proposition.

Thus we have obtained a subdivision of each connected component of $\mathfrak{F}$,

$$
\mathfrak{F}^{(z)}=\bigcup_{\Sigma \in \mathscr{S}(z)} \mathfrak{F}_{\Sigma}
$$

into parts that are homeomorphic to a Hilbert space, thanks to property (8). This is a generalization to flag varieties of the stratification in section 7.3 of [23]. Let $\sigma_{\Sigma}$, for each $\Sigma \in \mathscr{S}(z)$, be a permutation of $S$ such that $\underline{\sigma}_{\Sigma}\left(S_{i}\right)=\Sigma_{i}$, for all $i, 1 \leq i \leq m$. Then this decomposition of $\mathfrak{F}$ translates directly to the group $G L_{\text {res }}(H)$ and results in

Proposition 2.4.16. (Birkhoff decomposition.) Each connected component of the group $G L_{\mathrm{res}}(H)$ decomposes as

$$
G L_{\mathrm{res}}^{(z)} H=\bigcup_{\Sigma \in \mathscr{S}(z)} U_{0} \underline{\sigma}_{\Sigma} P .
$$

Remark 2.4.17. The decomposition derived here is the analytic equivalent of the algebraic decomposition from [22]. 


\section{§3. Holomorphic Line Bundles over $\mathfrak{F}^{(0)}$}

3.1. Another description of $\mathfrak{F}^{(0)}$. For each $\Sigma$ in $\mathscr{S}(z)$, there are numerous $\underline{\sigma} \in W$ such that $F_{\Sigma}=\underline{\sigma} F^{(0)}$. We start by describing a special choice that is useful at the description of $\mathfrak{F}^{(0)}$ as the homogeneous space of another group.

We construct a bijection $\sigma_{i}: S_{i} \rightarrow \Sigma_{i}$, as follows: since $S_{i}$ and $\Sigma_{i}$ are commensurable, there is a $N \geq 0$ such that

$$
\left\{s_{i}(k) \mid k>N\right\} \subset \Sigma_{i} \cap S_{i} .
$$

Consider the finite set $\Sigma_{i}-\left\{s_{i}(k) \mid k>N\right\}$. If it is empty, then we define $\sigma_{i}: S_{i} \rightarrow \Sigma_{i}$ as follows:

$$
\sigma_{i}\left(s_{i}(k)\right)=s_{i}(k+N) \quad \text { for all } k, 1 \leq k<m_{i}+1 .
$$

In this case we put $t_{i}=-N$.

If $\Sigma_{i}$ is not equal to $\left\{s_{i}(k) \mid k>N\right\}$, then we write $\Sigma_{i}-\left\{s_{i}(k) \mid k>N\right\}=$ $\left\{t_{1}, \ldots t_{N+\ell_{1}}\right\}$ for some $\ell_{i}$ in $\mathbf{Z}, t_{i}>-N$, and we define $\sigma_{i}$ by

$$
\begin{array}{ll}
\sigma_{i}\left(s_{i}(k)\right)=t_{k} & \text { for all } k, 1 \leq k \leq N+\ell_{i}, \\
\sigma_{i}\left(s_{i}(k)\right)=s_{i}\left(k-\ell_{i}\right) & \text { for all } k>N+\ell_{i} .
\end{array}
$$

For all $i, 1 \leq i \leq m$, consider the map $\tau_{i}: S_{i} \rightarrow S_{i}$ defined by

$$
\begin{array}{ll}
\tau_{i}\left(s_{i}(k)\right)=s_{i}(N+1) & \text { for all } k, 1 \leq k \leq N+\ell_{i} \\
\tau_{i}\left(s_{i}(k)\right)=s_{i}\left(k-\ell_{i}\right) & \text { for all } k>N+\ell_{i} .
\end{array}
$$

Since $p_{i} \circ \underline{\sigma}_{i}-\underline{\tau}_{i}$ is a finite dimensional operator, $p_{i} \circ \underline{\sigma}_{i}$ and $\underline{\tau}_{i}$ have the same index and for $\underline{\tau}_{i}$ one clearly has ind $\left(\underline{\tau}_{i}\right)=N+\ell_{i}-N=\ell_{i}$. In other words, the number $\ell_{i}$ is equal to $z_{i}$. The $\left\{\sigma_{i} \mid 1 \leq i \leq m\right\}$ compose to a bijection $\sigma: S \rightarrow S$ such that $\underline{\sigma} F^{(0)}=F_{\Sigma}$. We will introduce a special term for this type of permutations.

Definition 3.1.1. Let $\Sigma$ be a partition of $S$ in $\mathscr{S}(z)$. A permutation $\sigma$ of $S$ such that $\sigma\left(S_{i}\right)=\Sigma_{i}$ is called admissible of level $N$ if the following property holds

(i) For each $i$ and for all $k>N+z_{i}, \sigma\left(s_{i}(k)\right)=s_{i}\left(k-z_{i}\right)$.

One easily verifies that the collection of admissible permutations of $S$ of all levels forms a subgroup $W_{a}$ of $W$. It has a normal subgroup $W_{a}^{(0)}=W_{a}$ $\cap G L_{\mathrm{res}}^{(0)}(H)$ such that the quotient $W_{a} / W_{a}^{(0)}$ is isomorphic to $Z$. The elements of $W_{a}^{(0)}$ can be described in a direct concrete way. If $G$ is a finite subset of $S$ and if $\rho$ is a permutation of $G$, then we denote the extension of $\rho$ by the identity to a permutation of $S$, by $\tilde{\rho}$. Then we have 


$$
\begin{aligned}
W_{a}^{(0)} & =\lim _{N} W_{a}^{(0)}(N)=\lim _{\vec{N}}\left\{\underline{\sigma} \mid \underline{\sigma} \in W_{a}^{(0)} \text { is of level } N\right\} \\
& =\{\underline{\tilde{\rho}} \mid \rho \text { a permutation of } G, G \text { a finite subset of } S\} .
\end{aligned}
$$

Now we can introduce another group that acts transitively on $\mathfrak{F}^{(0)}$. Its advantage is that it enables you to construct in a simple way holomorphic line bundles over $\mathfrak{F}^{(0)}$. Let $\Sigma$ be in $\mathscr{S}(0)$ and let $\sigma$ be an admissible permutation of $S$ such that $\sigma\left(S_{i}\right)=\Sigma_{i}$. From the definition of admissibility we know that $\underline{\sigma}$ decomposes in operators $\left(\underline{\sigma}_{i j}\right)$ with the properties

(i) For each $1 \leq i \leq m, \underline{\sigma}_{i i}=\mathrm{Id}_{H_{l}}+$ a "finite-size" operator.

(ii) For all $i$ and $j, i \neq j, \underline{\sigma}_{i j}$ is a "finite-size" operator.

Since every flag $F$ in $\mathscr{F}^{(0)}$ is transversal to some $F_{\Sigma}$, with $\Sigma$ in $\mathscr{S}(0)$, we may conclude that each $F$ in $\mathfrak{F}^{(0)}$ is equal to $g \cdot F^{(0)}$ with $g \in G L_{\text {res }}(H)$ of the form

(a) For each $i, 1 \leq i \leq m, g_{i i}=\mathrm{Id}_{H_{1}}+$ a "finite-size" operator.

(b) For all $i$ and $j, i<j, g_{i j}$ is a "finite-size" operator.

(c) For all $i$ and $j, j<i, g_{i j}$ belongs to $\mathscr{H} \mathscr{S}\left(H_{j}, H_{i}\right)$.

Note that for all the operators $g_{i i}$ from (a) $\operatorname{det}\left(g_{i i}\right)$ is defined. Since we are working in an analytic setting we will consider a somewhat wider class of operators such that on one hand we work in a Banach framework and on the other we can take determinants of certain minors. Recall, see [12], that the determinant is defined for each operator of the form "identity $+a$ nuclear operator". Therefore we introduce

$$
B_{2}(H)=\left\{g \mid g \in \mathscr{B}_{\text {res }}(H), \quad \begin{array}{ll}
g_{i i}-\operatorname{Id}_{H_{1}} \in \mathscr{I}\left(H_{i}\right) \\
g_{i j} \in \mathscr{H} \mathscr{P}\left(H_{j}, H_{i}\right) \text { for } i \neq j
\end{array}\right\} .
$$

On $B_{2}(H)$ we put a different topology than the one induced by $\mathscr{B}_{\text {res }}(H)$. For, let $\mathscr{Z}$ be the subspace of $\mathscr{B}_{\text {res }}(H)$ defined by

$$
\mathscr{Z}=\left\{b \mid b \in \mathscr{B}_{\mathrm{res}}(H), \quad \begin{array}{l}
b_{i j} \in \mathscr{H} \mathscr{S}\left(H_{j}, H_{i}\right) \text { for } i \neq j \\
b_{i i} \in \mathcal{A}^{\wedge}\left(H_{i}\right)
\end{array}\right\} .
$$

Then $\mathscr{Z}$ is a Banach space if we equip it with the norm $\|\cdot\|_{\mathscr{L}}$ given by

$$
\|b\|_{\mathscr{Z}}=\sum_{i \neq j}\left\|b_{i j}\right\|_{\mathscr{H} \mathscr{Y}}+\sum_{i=1}^{m}\left\|b_{i i}\right\|_{1} .
$$

The collection $B_{2}(H)$ is nothing but $\mathscr{Z}$ shifted by the identity and we transfer the Banach structure on $\mathscr{Z}$ to $B_{2}(H)$ by means of the map $g \mapsto g+$ Id. Since the product of two Hilbert-Schmidt operators is nuclear, one sees that $B_{2}(H)$ is closed under multiplication. Moreover the multiplication with an element of $B_{2}(H)$ is an analytic map from $B_{2}(H)$ to itself. In $B_{2}(H)$ we have the subgroup $U_{-}$and its "adjoint" the group 


$$
U_{+}=\left\{u^{*} \mid u \in U_{-}\right\} \text {. }
$$

Consider an element $b$ in $B_{2}(H)$. Now we define $u=\left(u_{i j}\right)$ in $U_{-}$and $v=\left(v_{i j}\right)$ in $U_{+}$by

$$
\begin{aligned}
& u_{i i}=v_{i i}=\operatorname{Id}_{H_{2}}, u_{i j}=-b_{i j} \text { if } i>j, u_{i j}=0 \text { if } j>i, \\
& v_{i j}=-b_{i j} \text { if } i<j \text { and } v_{i j}=0 \text { if } i>j .
\end{aligned}
$$

A direct verification shows that $u b v$ belongs to $\operatorname{Id}+\mathscr{N}(H)$. Since $B_{2}(H)$ is closed w.r.t. taking adjoints, we have

Lemma 3.1.2. Every $b \in B_{2}(H)$ can be written in the form $b=u_{1} b_{1} v_{1}$ or $b=v_{2} b_{2} u_{2}$, where $u_{1}$ and $u_{2}$ belong to $U_{-}, v_{2}$ and $v_{1}$ belong to $U_{+}$and $b_{1}$ and $b_{2}$ lie in $\mathrm{Id}+\mathscr{N}(H)$.

The decompositions in lemma 3.1 .2 are clearly not unique, but they suffice to define a determinant map det: $B_{2}(H) \rightarrow$ C. Namely, for $b=u_{1} b_{1} v_{1}$ as in lemma 3.1.2, we put $\operatorname{det}(b)=\operatorname{det}\left(u_{1} b_{1} v_{1}\right)=\operatorname{det}\left(b_{1}\right)$.

To see that this is well-defined, we note first of all that for $u \in U_{-}$? $\{\operatorname{Id}+\mathcal{N}(H)\}$ and $v \in U_{+} \cap\{\operatorname{Id}+\mathcal{N}(H)\}$ we have $\operatorname{det}(u)=\operatorname{det}(v)=1$, since $u$ - Id and $v$ - Id have zero trace. Now, assume $b \in B_{2}(H)$ can be written as $b=u_{1} b_{1} v_{1}=u_{2} b_{2} v_{2}$ with $b_{i} \in \mathrm{Id}+\mathcal{N}(H), u_{i} \in U_{-}$and $v_{i} \in U_{+}$. Then $b_{2}=$ $\left(u_{2}^{-1} u_{1}\right) b_{1}\left(v_{1} v_{2}^{-1}\right)$ and, since both $b_{1}$ and $b_{2}$ belong to $\mathrm{Id}+\mathscr{N}(H)$, this implies that $u_{2}^{-1} u_{1} \in U_{-} \cap\{\operatorname{Id}+\mathscr{N}(H)\}$ and $v_{1} v_{2}^{-1} \in U_{+} \cap\{\operatorname{Id}+\mathscr{N}(H)\}$. By the multiplicativity of the determinant on $\operatorname{Id}+\mathscr{N}(H)$, we get $\operatorname{det}\left(b_{2}\right)=\operatorname{det}\left(u_{2}^{-1} u_{1}\right)$ $\operatorname{det}\left(b_{1}\right) \operatorname{det}\left(v_{1} v_{2}^{-1}\right)=\operatorname{det}\left(b_{1}\right)$.

Remark 3.1.3. Since the operators in Id $+\mathscr{N}(H)$ lie dense in $B_{2}(H)$ and since det is multiplicative on Id $+\mathscr{N}(H)$ we get that for each $b_{1}$ and $b_{2}$ in $B_{2}(H)$

$$
\operatorname{det}\left(b_{1} b_{2}\right)=\operatorname{det}\left(b_{1}\right) \operatorname{det}\left(b_{2}\right)
$$

From the fact that an operator $g$ of the form $\mathrm{Id}+\mathcal{N}(H)$ is invertible if and only if $\operatorname{det}(g)$ is non-zero, we see that the invertible elements of $B_{2}(H)$ form a group $(\mathfrak{5}$ and are given by

$$
\mathfrak{G}=\left\{b \mid b \in B_{2}(H), \operatorname{det}(b) \neq 0\right\} .
$$

Clearly $\mathfrak{6}$ is a Banach Lie group with Lie algebra $\mathscr{Z}$ and it acts analytically and transitively on $\mathfrak{F}^{(0)}$. The stabilizer of $F^{(0)}$ in $(\mathfrak{5}$ has the form

$$
\mathscr{T}=\left\{t=\left(\begin{array}{cccc}
t_{11} & \cdots & & t_{1 m} \\
0 & & \vdots \\
\vdots & \ddots & & \vdots \\
0 & \cdots & 0 & t_{m m}
\end{array}\right) \begin{array}{c}
t_{i i} \in\left\{\operatorname{Id}+\mathscr{N}^{\prime}\left(H_{i}\right)\right\} \cap G L\left(H_{i}\right) \\
t_{i j} \in \mathscr{H} \mathscr{S}\left(H_{j}, H_{i}\right) \text { for } j>i
\end{array}\right\}
$$


Thus we can identity $\mathfrak{F}^{(0)}$ with the homogeneous space $\mathfrak{5} / \mathscr{T}$.

Remark 3.1.4. If we would work with $\mathfrak{F}(\mathscr{N})$ instead of $\mathfrak{F}$, then we could simply take instead of $B_{2}(H)$ the collection $\mathrm{Id}+\mathscr{N}(H)$ and instead of $\mathfrak{F}$ the group of invertible operators of the form $\mathrm{Id}+\mathscr{N}(H)$. If one likes to work with $\mathfrak{F}(\mathscr{C})$ then the group

$$
G_{\mathscr{C}}=\left\{b \mid b=\left(b_{i j}\right) \in G L(H), \quad \begin{array}{ll} 
& b_{i j} \in \mathscr{N}\left(H_{j}, H_{i}\right) \text { if } j>i \\
& b_{i j} \in \mathscr{C}\left(H_{j}, H_{i}\right) \text { if } j<i
\end{array}\right\}
$$

acts transitively on the connected component of $\mathfrak{F}(\mathscr{C})$ containing the basic flag and allows you to take determinants of suitable minors.

3.1.5. Next we consider the maximal torus $T(\mathscr{N})=T \cap \mathfrak{b}$ in $\mathfrak{6}$. It consists of all operators of the form $\operatorname{diag}\left(\left\{1+t_{s}\right\}\right)$, with $1+t_{s} \neq 0$ and $\sum_{s \in S}\left|t_{s}\right|<\infty$. In $T(\mathcal{N})$ we have the dense subgroup $T_{f}$ given by

$$
T_{f}=\left\{t \mid t=\operatorname{diag}\left(\left\{1+t_{s}\right\}\right) \in T(\mathscr{N}), t_{s} \neq 0 \text { for only finitely many } s \text { in } S\right\}
$$

Any analytic group homomorphism of $T_{f}$ into $\mathbf{C}^{*}$ has the form

$$
t=\operatorname{diag}\left(\left(1+t_{s}\right)\right) \longmapsto \prod_{s \in S}\left(1+t_{s}\right)^{m_{s}}=: \chi_{\underline{m}}(t),
$$

where $\underline{m}=\left\{m_{s}\right\}$, with $m_{s} \in \mathbf{Z}$ for all $s \in S$. This character $\chi_{\underline{m}}$ can be continued to an analytic character of $T(\mathcal{N})$ if and only if there are only finitely many different $m_{s}, s \in S$. This extension of $\chi_{\underline{\underline{m}}}$ is also denoted by $\chi_{\underline{m}}$ and we write $\hat{T}$ for the group of analytic characters of $T\left(\mathcal{N}^{n}\right)$. Following the finite dimensional terminology, we will speak, when $T(\mathcal{N})$ acts on a vector according to a $\chi \in \hat{T}$, of " $v$ is a vector of weight $\chi$ ".

For each $s$ and $r$ in $S$, let $E_{s r}$ be the operator in $\mathscr{B}_{\text {res }}(H)$ given by

$$
E_{\mathrm{sr}}\left(e_{l}\right)=\delta_{r l} e_{\mathrm{s}} \quad \text { for all } l \in S .
$$

The adjoint action of $T(\mathcal{N})$ on $\mathscr{B}_{\text {res }}(H)$ gives for these elements

$$
t E_{s r} t^{-1}=\frac{1+t_{s}}{1+t_{r}} E_{s r}=: \chi_{s r}(t) E_{s r} .
$$

A character $\chi$ of $T(\mathcal{N})$ is called positive, notation $\chi \geq 0$, if it belongs to the semigroup generated by the $\left\{\chi_{s r} \mid s \in S_{i}, r \in S_{j}, i>j\right\}$. This enables you to define a partial order on $\hat{T}$ by

$$
\chi \leq \psi \Longleftrightarrow 0 \leq \chi^{-1} \psi
$$

Clearly we call $\chi$ in $\hat{T}$ negative if and only if $\chi^{-1} \geq 0$. 
Remark 3.1.6. One can see the space $\mathfrak{F}^{(0)}$ also as a homogeneous space for the group of invertible operators in "Id $+\mathscr{H} \mathscr{S}(H)$ ", which in its turn can be identified with an open part of the Hilbert-Schmidt operators on $H$. This group, however, does not permit you to take suitable minors.

Remark 3.1.7. One can give the same type of description for the other components $\mathfrak{F}^{(z)}$ by taking some $\Sigma \in \mathscr{S}(z)$ and by introducing the group $\mathfrak{b}$ as the operators that decompose w.r.t. $H=H_{\Sigma_{1}} \oplus \cdots \oplus H_{\Sigma_{m}}$ in the above way.

3.2. Finite dimensional subvarieties. In this subsection we consider some finite dimensional flag subvarieties contained in $\mathfrak{F}^{(0)}$. Let $K$ be a finite subset of $S$. For simplicity we assume that $K$ contains all the $S_{i}$ that are finite. The general linear group $G L\left(H_{K}\right)$ embeds into $G L_{\text {res }}^{(0)}(H)$ by extending $u \in G L\left(H_{K}\right)$ on $H_{S-K}$ by the identity. We write $\mathfrak{F}_{K}$ for the subvariety of $\mathfrak{F}^{(0)}$ given by

$$
\mathfrak{F}_{K}=\left\{u F^{(0)} \mid u \in G L\left(H_{K}\right)\right\}=\left\{u F^{(0)} \mid u \in U\left(H_{K}\right)\right\} .
$$

If $K_{1} \subset K_{2}$, then we have a natural embedding of $G L\left(H_{K_{1}}\right)$ into $G L\left(H_{K_{2}}\right)$ and of $\mathfrak{F}_{K_{1}}$ in $\mathfrak{F}_{K_{2}}$. Now one considers a collection of finite subsets $\left\{K_{n} \mid n \in \mathbf{N}\right\}$ of $S$ given by

$$
K_{n}=\bigcup_{i=1}^{m}\left\{s_{i}(k) \mid k \leq n+\max _{m_{J}<\infty}\left(m_{j}\right)\right\}
$$

Then $S=\bigcup_{n \in \mathbf{N}} K_{n}$ and with the identifications mentioned above we write

$$
G L(\infty)=\bigcup_{n \in \mathbf{N}} G L\left(H_{K_{n}}\right), U(\infty)=\bigcup_{n \in \mathbf{N}} U\left(H_{K_{n}}\right) \text { and } \mathfrak{F}(\infty)=\bigcup_{n \in \mathbf{N}} \mathfrak{F}_{K_{n}}
$$

Since, for all $i \neq j$, the "finite-size" operators are dense in $\mathscr{H} \mathscr{S}\left(H_{j}, H_{i}\right)$, and the $U_{\Sigma}, \Sigma \in \mathscr{S}(0)$, cover $\mathfrak{F}^{(0)}$, we get

Lemma 3.2.1. The space $\mathfrak{F}(\infty)$ lies dense in $\mathfrak{F}^{(0)}$.

Consider now a holomorphic function $f$ on $\mathfrak{F}^{(0)}$. The restriction of $f$ to some $\mathfrak{F}_{K_{n}}$ must be a constant, since $\mathfrak{F}_{K_{n}}$ is a compact complex manifold. Hence $f$ is a constant on $\mathfrak{F}(\infty)$ and the lemma implies then

Corollary 3.2.2. The only holomorphic functions from $\mathfrak{F}^{(0)}$ to $\mathrm{C}$ are the constants.

This is a generalization for flag varieties of Proposition 7.2.2 in [23].

Remark 3.2.3. The results of this subsection remain true if one would work with the nuclear flag space $\mathfrak{F}(\mathcal{N})$ or the "compact" flag variety $\mathfrak{F}(\mathscr{C})$. However the "finite-size" flags from $\mathfrak{F}(\infty)$ are not lying dense in the space of bounded flags $\mathfrak{F}(\mathscr{B})$. 
3.3. The line bundles and the central extension. For each $\underline{k}=\left(k_{1}, \ldots, k_{m}\right)$ in $\mathbf{Z}^{m}$, we define $\psi_{\underline{k}}$ in $\hat{T}$ by

$$
\psi_{\underline{k}}\left(\operatorname{diag}\left\{1+t_{s}\right\}\right)=\prod_{s_{1} \in S_{1}}\left(1+t_{s_{1}}\right)^{k_{1}} \prod_{s_{2} \in S_{2}}\left(1+t_{s_{2}}\right)^{k_{2}} \cdots \prod_{s_{m} \in S_{m}}\left(1+t_{s_{m}}\right)^{k_{m}}
$$

Clearly $\psi_{\underline{k}}$ extends to an analytic character of $\mathscr{T}$ by means of the formula

$$
\psi_{\underline{k}}(t)=\operatorname{det}\left(t_{11}\right)^{k_{1}} \cdots \operatorname{det}\left(t_{m m}\right)^{k_{m}} .
$$

According to section 6.5 in [4], there exists for each analytic character $\psi_{\underline{k}}$ of $\mathscr{T}$, a holomorphic line bundle $L(\underline{k})=\left(\mathfrak{H} \otimes^{\mathscr{T}} \mathbf{C}\right.$ over $\mathscr{F}^{(0)}=(\mathfrak{5} / \mathscr{T}$. It is concretely defined as follows: consider on the space $\mathfrak{G} \times \mathbf{C}$ the equivalence relation

$$
\left(g_{1}, \lambda_{1}\right) \sim\left(g_{2}, \lambda_{2}\right) \Longleftrightarrow g_{1}=g_{2} \circ t, \text { with } t \in \mathscr{T} \text { and } \lambda_{2}=\lambda_{1} \psi_{\underline{k}}(t) .
$$

The space $(\mathfrak{5} \times \mathbf{C}$ modulo this equivalence relation is $L(\underline{k})$. For each $g \in \mathfrak{b}$ and each $\lambda$ in $\mathbf{C}$, we denote the equivalence class to which the pair $(g, \lambda)$ belongs by $[g, \lambda]$. There is a natural projection $\pi_{\underline{k}}: L(\underline{k}) \rightarrow \mathfrak{F}^{(0)}$ given by

$$
\pi_{\underline{k}}([g, \lambda])=g \cdot F^{(0)} .
$$

The space $L(\underline{k})$ is a Hilbert manifold based on the Hilbert space $E \oplus \mathbf{C}$. For each $\Sigma \in \mathscr{S}(0)$ one can give a concrete trivialization of $L(\underline{k})$ above $U_{\Sigma}$. Let $\sigma$ be an admissible permutation of $S$ such, that $\Sigma_{i}=\sigma\left(S_{i}\right)$. Then we define $\varphi_{\Sigma}: E \oplus \mathbf{C} \rightarrow \pi_{\underline{k}}^{-1}\left(U_{\Sigma}\right)$ by

$$
\varphi_{\Sigma}(A, \lambda)=[\underline{\sigma}(\mathrm{Id}+A), \lambda] .
$$

Assume we have a $\Sigma$ and $R$ in $\mathscr{S}(0)$ such that $\pi_{\underline{k}}^{-1}\left(U_{\Sigma}\right) \cap \pi_{\underline{k}}^{-1}\left(U_{R}\right)$ is non-empty. Let $\sigma$ and $\rho$ be admissible permutations of $S$ with $\sigma\left(S_{i}\right)=\Sigma_{i}$ and $\rho\left(S_{i}\right)=R_{i}$. If $(A, \lambda)$ is such that $\varphi_{\Sigma}(A, \lambda)$ belongs to $\pi_{\underline{k}}^{-1}\left(U_{R}\right)$ then we know that $\underline{\rho}^{-1} \underline{\sigma}(\mathrm{Id}+A)$ belongs to $\Omega \cap(\mathfrak{5}$ and because of Lemma 2.2.1

$$
\underline{\rho}^{-1} \underline{\sigma}(\operatorname{Id}+A)=u(A) p(A), \text { with } u(A) \in U_{-}, p(A) \in \mathscr{T}
$$

Here $u(A)$ and $p(A)$ depend analytically of $A$ and thus we get

$$
\varphi_{R}^{-1} \varphi_{\Sigma}(A, \lambda)=\left(u(A)-\mathrm{Id}, \lambda \psi_{\underline{k}}(p(A))\right)
$$

and this map is clearly analytic. This proves that

Lemma 3.3.1. The $\left\{\left(\pi_{\underline{k}}^{-1}\left(U_{\Sigma}\right), \varphi_{\Sigma}^{-1}\right) \mid \Sigma \in \mathscr{S}(0)\right\}$ are the charts of an analytic $E \oplus \mathbf{C}_{-}$structure on $L(\underline{k})$. 
Remark 3.3.2. For the case $m=2, m_{1}=m_{2}=\infty$, the bundles $L((+1,0))$ and $L((-1,0))$ are the determinant bundle Det and its dual Det* as introduced in section 7.7 of [23].

There is a natural analytic action of the group $(\mathfrak{5}$ on the space $L(\underline{k})$ by left translations

$$
g_{1} \cdot\left[g_{2}, \lambda\right]=\left[g_{1} g_{2}, \lambda\right]
$$

This is a lifting of the natural action of $\mathfrak{F}$ on $\mathfrak{F}^{(0)}$ to one on $L(\underline{k})$. However, the natural action of $G L_{\text {res }}^{(0)}(H)$ can, in general, not be lifted to one on $L(\underline{k})$. Such an attempt may lead to nontrivial central extensions of $G L_{\text {res }}^{(0)}(H)$ as we will show.

Note that each $g$ in $G L_{\text {res }}^{(0)}(H)$ can be written as $g=d g_{2}$, with $g_{2} \in \mathfrak{5}$ and $d$ belonging to the subgroup

$$
D=\left\{g \mid g=\left(g_{i j}\right) \in G L_{\mathrm{res}}^{(0)}(H), g_{i j}=0 \text { if } i \neq j\right\} .
$$

of $G L_{\text {res }}^{(0)}(H)$. Clearly the group $D$ normalizes the group $(5$. Since the determinant of an operator of the form "identity + nuclear" is invariant under conjugation with an invertible operator, we get that $D$ centralizes each $\psi_{\underline{k}}$, i.e. for each $t$ in $\mathscr{T}$ and each $d$ in $D$ we have

$$
\psi_{\underline{k}}\left(d t d^{-1}\right)=\psi_{\underline{k}}(t) .
$$

This fact permits you to lift the action of $D$ on $\mathfrak{F}^{(0)}$ to one on $L(\underline{k})$ by means of

$$
d \cdot[g, \lambda]=\left[d g d^{-1}, \lambda\right] .
$$

For an element $d$ from $D \cap \mathfrak{G}$, this action differs by a factor $\psi_{\underline{k}}\left(d^{-1}\right)$ from the action induced by that of $\mathbf{6}$. Hence we cannot combine them to an action of $G L_{\text {res }}^{(0)}(H)$ on $\mathfrak{F}^{(0)}$. To overcome this problem we build a group extension $G$ of $G L_{\text {res }}^{(0)}(H)$. It is defined by

$$
G=\left\{(g, d) \mid g \in G L_{\mathrm{res}}^{(0)}(H), d \in D \text { and } g d^{-1} \in \mathfrak{( b}\right\} .
$$

As one verifies directly this group acts on $L(\underline{k})$ by means of

$$
(g, d)\left[g_{1}, \lambda_{1}\right]=\left[g g_{1} d^{-1}, \lambda_{1}\right]
$$

It is simply the combination of the $(\mathfrak{H}$-action and the $D$-action given above. Let $\pi: G \rightarrow G L_{\mathrm{res}}^{(0)}(H)$ be the canonical projection, i.e. $\pi((g, d))=g$ for all $(g, d) \in G$. For certain subgroups of $G L_{\text {res }}^{(0)}(H)$ there exist several ways to embed them into $G$. Therefore we introduce special notations for two of them. Let $\underline{i}$ resp. 
$\underline{j}$ be the embedding of $(5$ resp. $D$ into $G$ given by

$$
\underline{i}(g)=(g, \mathrm{Id}) \text { and } \underline{j}(d)=(d, d) .
$$

As a group $G$ is the semi-direct product of $\underline{i}(\mathbf{6})$ and $j(D)$. We equip each $G L\left(H_{i}\right)$ with the operator norm topology and we put on $j(D)$ the product Banach Lie group structure. On $\underline{i}(\mathfrak{G})$ we take the Banach structure based on $\mathscr{Z}$. The conjugation with an element $d$ of $D$ defines an analytic diffeomorphism of $\mathfrak{b}$. Hence if we put on $G$ the product topology of $\underline{i}(\mathfrak{5})$ and $j(D)$, it becomes a Banach Lie group based on

$$
\left(\bigoplus_{i=1}^{m} \mathscr{B}\left(H_{i}\right)\right) \oplus \mathscr{Z} .
$$

The group $G$ is a fiber bundle over $G L_{\text {res }}^{(0)}(H)$, with fiber $\mathscr{T} \cap D$. This is clear from the following useful trivializations. For each $\Sigma=\left(\Sigma_{i}\right)$ in $\mathscr{S}(0)$ consider the open set $G(\Sigma)$ of $G$ given by

$$
G(\Sigma)=\left\{(g, d)\left|(g, d) \in G, p_{\Sigma_{i}} \circ g\right| H_{i} \text { is a bijection for all } i, 1 \leq i \leq m\right\} .
$$

The group $G$ is the union of these open sets. If $\sigma$ is an admissible permutation with $\sigma\left(S_{i}\right)=\Sigma_{i}$, then we define an analytic bijection from $\pi(G(\Sigma)) \times\{\mathscr{T} \cap D\}$ to $G(\Sigma)$ by

$$
(g, t) \longmapsto(g, \tilde{d})(\mathrm{Id}, t),
$$

where $\tilde{d}$ in $D$ is determined by

$$
\tilde{d}_{i i}=\underline{\sigma}^{-1} \circ p_{\Sigma_{i}} \circ g \mid H_{i}
$$

Next we try to minimalize the extension of $G L_{\text {res }}^{(0)}(H)$ that acts on $\mathfrak{F}^{(0)}$ and $L(\underline{k})$. Thereto we consider the action of the kernel of $\pi$ on $L(\underline{k})$

$$
(\mathrm{Id}, d) \cdot[g, \lambda]=\left[g d^{-1}, \lambda\right]=\left[g, \psi_{\underline{k}}\left(d^{-1}\right) \lambda\right] .
$$

In particular the group $D(\underline{k})=\left\{(\operatorname{Id}, d) \mid(\operatorname{Id}, d) \in G\right.$ and $\left.\psi_{\underline{k}}(d)=1\right\}$ acts trivially on $L(\underline{k})$ and we see that it suffices to consider the extension $G(\underline{k})=G / D(\underline{k})$ of $G L_{\mathrm{res}}^{(0)}(H)$. If the character $\psi_{\underline{k}}$ is trivial, i.e. $\underline{k}=0$, then $G(\underline{k})$ is just $G L_{\mathrm{res}}^{(0)}(H)$. For $\underline{k} \neq 0$, one computes directly that $G(\underline{k})$ is a central extension of $G L_{\mathrm{res}}^{(0)}(H)$ with $\operatorname{Ker}(\pi) / D(\underline{k}) \cong \mathbf{C}^{*}$. For $m=2, m_{1}=m_{2}=\infty$, the extension $G((-1,0))$ is the one introduced in section 6.6 of [23].

One can describe such an extension with a Borel 2-cocycle $\alpha: G L_{\text {res }}^{(0)}(H) \times$ $G L_{\text {res }}^{(0)}(H) \rightarrow C^{*}$. It can be constructed as follows: take a section $\rho$ of the fiber bundle $G \stackrel{\pi}{\rightarrow} G L_{\text {res }}^{(0)}(H)$, i.e. for each $g$ in $G L_{\text {res }}^{(0)}(H)$ we have 


$$
\rho(g)=(g, q(g)) \text { with } q(g) \in D .
$$

By definition we have for each $g_{1}$ and $g_{2}$ in $G L_{\text {res }}^{(0)}(H)$ that

$$
q\left(g_{1}\right) q\left(g_{2}\right) q\left(g_{1} g_{2}\right)^{-1} \in D \cap \mathfrak{G} \text {. }
$$

Thus we get for the action on $L(\underline{k})$ the relation

$$
\begin{aligned}
\rho\left(g_{1} g_{2}\right) \cdot[g, \lambda] & =\rho\left(g_{1}\right) \cdot\left\{\rho\left(g_{2}\right) \cdot\left[g, \lambda \psi_{\underline{k}}\left(q\left(g_{1}\right) q\left(g_{2}\right) q\left(g_{1} g_{2}\right)^{-1}\right)\right]\right\} \\
& =: \rho\left(g_{1}\right) \cdot\left\{\rho\left(g_{2}\right) \cdot\left[g, \lambda \alpha\left(g_{1}, g_{2}\right)^{-1}\right]\right\} .
\end{aligned}
$$

The group $G(\underline{k})$ is then isomorphic as a group to the product space $G L_{\mathrm{res}}^{(0)}(H) \times \mathrm{C}^{*}$ with the multiplication

$$
\left(g_{1}, \lambda_{1}\right) *\left(g_{2}, \lambda_{2}\right)=\left(g_{1} g_{2}, \lambda_{1} \lambda_{2} \alpha\left(g_{1}, g_{2}\right)\right)
$$

If $\tilde{\rho}$ is another section of $G \stackrel{\pi}{\rightarrow} G L_{\text {res }}^{(0)}(H)$ with $\tilde{\rho}(g)=(g, \tilde{q}(g))$, then we have by definition for each $g$ in $G L_{\text {res }}^{(0)}(H)$ that $\tilde{q}(g)=q(g) t(g)$ with $t(g)$ in $\mathscr{T} \cap D$. The corresponding 2-cocycle $\tilde{\alpha}$ satisfies

$$
\tilde{\alpha}\left(g_{1}, g_{2}\right)=\frac{\psi_{\underline{k}}\left(t\left(g_{1} g_{2}\right)\right)}{\psi_{\underline{k}}\left(t\left(g_{1}\right)\right) \psi_{\underline{k}}\left(t\left(g_{2}\right)\right)} \quad \alpha\left(g_{1}, g_{2}\right) .
$$

In other words, it differs by a trivial 2-cocycle and we merely have to consider one section $\rho$.

A section $\rho$ can be composed from the local trivializations of $\pi: G \rightarrow$ $G L_{\text {res }}^{(0)}(H)$ defined above. First we number the elements of $\mathscr{S}(0): \mathscr{S}(0)=\left\{\Sigma^{(i)} \mid\right.$ $i \geq 0\}$, such that $\Sigma^{(0)}$ is the partition corresponding to the basic flag. For $g \in G\left(\Sigma^{(0)}\right)$ we choose $q(g)$ according to the trivialization (9) with $\sigma=$ Id. Next we define $q(g)$ inductively by: if $g$ belongs to $\bigcup_{i=0}^{m} G\left(\Sigma^{(i)}\right)$ and not to $\bigcup_{i=0}^{m-1} G\left(\Sigma^{(i)}\right)$, then we take $q(g)$ according to the trivialization of $G\left(\Sigma^{(m)}\right)$ given by (9). In particular if $g, h$ and $g h$ belong to $G\left(\Sigma^{(0)}\right)$, then the 2-cocycle $\alpha$ is given by

$$
\alpha(g, h)=\prod_{i=1}^{m} \operatorname{det}\left(\mathrm{Id}+\sum_{j \neq i} g_{i j} h_{j i} h_{i i}^{-1} g_{i i}^{-1}\right)^{k_{i}} .
$$

From this formula we will compute in the next subsection the corresponding Lie algebra 2-cocycle.

3.4. The non-triviality of the extension $G(\underline{k})$. First we consider the case that $k_{i}=l, l \in \mathbf{Z}$, for all $i$. Then we have for each $g \in D \cap \mathscr{T}$ that $\psi_{\underline{k}}(g)=\operatorname{det}(g)^{l}$. We can adjust the $(\mathfrak{5}$-action on $L(\underline{k})$ as follows:

$$
g *[x, \lambda]=\left[g x, \operatorname{det}(g)^{-l} \lambda\right] .
$$


Hence for elements $d \in D \cap \mathfrak{5}$, we get

$$
d *[x, \lambda]=\psi_{\underline{k}}\left(d^{-1}\right)\left[d x d^{-1} d, \lambda\right]=\left[d x d^{-1}, \lambda\right] .
$$

Now we combine this new $\left(\mathfrak{5}\right.$-action with that of $D$ and we define for $g=d_{1} g_{1}$ in $G L_{\mathrm{res}}^{(0)}(H)$, where $d_{1} \in D$ and $g-1 \in \mathfrak{6}$,

$$
g *[x, \lambda]:=\left[d_{1} g_{1} x d_{1}^{-1}, \operatorname{det}\left(g_{1}\right)^{-l} \lambda\right] .
$$

It is a straightforward verification to show that this is well-defined and that it defines an action of $G L_{\mathrm{res}}^{(0)}(H)$ on $L(\underline{k})$. This implies that $G(\underline{k})$ is a trivial central extension of $G L_{\text {res }}^{(0)}(H)$.

Secondly we consider the case where at most one of the $m_{i}$ is infinite. Then $G L_{\text {res }}^{(0)}(H)$ is simply $G L(H)$ and we know from [20] that this group is contractible. In particular the fiber bundle $G \stackrel{\pi}{\rightarrow} G L(H)$ is then topologically trivial and the group $G(\underline{k})$ is the direct product of $G L(H)$ and $\operatorname{Ker}(\pi) / D(\underline{k})$. Hence we may assume in the sequel that there are at least two infinite $m_{i}$ 's.

The next case we have a look at is that $\underline{k}$ satisfies

$$
k_{i} \neq 0 \Longrightarrow m_{i}<\infty
$$

Let $g \mapsto(g, q(g))$ be a section of $G \rightarrow G L_{\text {res }}^{(0)}(H)$. We will adjust $q(g)$ such that the 2-cocycle determining $G(\underline{k})$ becomes trivial. Namely, we define $\tilde{q}(g)$ in $D$ by

$$
\begin{aligned}
& \tilde{q}(g)_{i i}=q(g)_{i i} \text { if } m_{i}=\infty \text { and } \\
& \tilde{q}(g)_{i i}=\operatorname{diag}\left(1, \ldots, 1, \operatorname{det}\left(q(g)_{i i}^{-1}\right)\right) q(g)_{i i}, \text { if } m_{i}<\infty
\end{aligned}
$$

Then $g \mapsto(g, \tilde{q}(g))$ defines another section $\tilde{\rho}$ of $G \rightarrow G L_{\mathrm{res}}^{(0)}(H)$ and the corresponding 2-cocycle $\tilde{\alpha}$ is trivial

$$
\tilde{\alpha}\left(g_{1}, g_{2}\right)=\psi_{\underline{k}}\left(\tilde{q}\left(g_{1} g_{2}\right) \tilde{q}\left(g_{2}\right)^{-1} \tilde{q}\left(g_{1}\right)^{-1}\right)=1 .
$$

In the cases considered so far we have seen that the fiber bundle $G \stackrel{\pi}{\rightarrow} G L_{\mathrm{res}}^{(0)}(H)$ is trivial and hence also the central extension $G(\underline{k})$ of $G L_{\mathrm{res}}^{(0)}(H)$. We will show now that the extension $G(\underline{k})$ can be non-trivial.

Note that the 2-cocycle $\alpha$ is given close to the identity by an analytic expression. Hence we can consider the corresponding Lie algebra 2-cocycle $d \alpha$. We consider the elements of $\mathscr{B}_{\text {res }}(H)$ as left invariant vector field on $G L_{\text {res }}^{(0)}(H)$. Then $d \alpha: \mathscr{B}_{\text {res }}(H) \times \mathscr{B}_{\text {res }}(H) \rightarrow \mathbf{C}$ is given by

$$
d \alpha(X, Y)=\left.\frac{d}{d t} \frac{d}{d s} \alpha(\exp (t X), \exp (s Y))\right|_{\substack{t=0 \\ s=0}}-\left.\frac{d}{d s} \frac{d}{d t} \alpha(\exp (s Y), \exp (t X))\right|_{\substack{t=0 \\ s=0}} .
$$

For $X=\left(X_{i j}\right)$ in $\mathscr{B}_{\text {res }}(H)$, we write $g=\exp (t X)=\left(g_{i j}\right)$. With respect to the 
parameter $t$ we have

$$
\begin{aligned}
& g_{i i}=\operatorname{Id}_{H_{i}}+t X_{i i}+\text { "higher order in } t " \\
& g_{i j}=t X_{i j}+\text { "higher order in } t " \text { for } i \neq j .
\end{aligned}
$$

If $h=\left(h_{i j}\right)=\exp (s Y)$, then we are interested in the $t s$-term in

$$
\begin{aligned}
& \operatorname{det}\left(\operatorname{Id}_{H_{i}}+\sum_{j \neq i} g_{i j} h_{j i} h_{i i}^{-1} g_{i i}^{-1}\right) \\
& \quad=\operatorname{det}\left(\operatorname{Id}_{H_{\imath}}+N\right)=1+\sum_{k=1}^{\infty} \operatorname{Trace}\left(\Lambda^{k} N\right) \\
& \quad=1+t s \sum_{j \neq i} \operatorname{Trace}\left(X_{i j} Y_{j i}\right)+\text { “at least } 2 \text { nd order in } t \text { or } s "
\end{aligned}
$$

By combining this expression with the local formula (10) for $\alpha$, we obtain the following formula for $d x$ :

$$
d \alpha(X, Y)=\sum_{i=1}^{m} k_{i} \text { Trace }\left\{\sum_{j \neq i} X_{i j} Y_{j i}-\sum_{j \neq i} Y_{i j} X_{j i}\right\} .
$$

This Lie algebra cocycle is trivial if it has the form $f([X, Y])$ with $f: \mathscr{B}_{\text {res }}(H) \rightarrow \mathbf{C}$ some linear map. The element $[X, Y]$ in $\mathscr{B}_{\text {res }}(H)$ has the form

$$
[X, Y]=\left(\begin{array}{cc}
{\left[X_{11}, Y_{11}\right]+\Sigma_{j \neq 1} X_{1 j} Y_{j 1}-\Sigma_{j \neq 1} Y_{1 j} X_{j 1}} & * \\
\ddots & \\
* & {\left[X_{m m}, Y_{m m}\right]+\Sigma_{j \neq m} X_{m j} Y_{j m}-\Sigma_{j \neq m} Y_{m j} X_{j m}}
\end{array}\right) .
$$

Note that if $\underline{k}$ satisfies (11), then we can directly define such an $f$. For, in that case, we have for all $i$ with $k_{i} \neq 0$ that Trace $\left[X_{i i}, Y_{i i}\right]$ is well-defined and equal to zero and we can take

$$
f(X)=\sum_{i, k_{\imath} \neq 0} k_{i} \text { Trace }\left(X_{i i}\right)
$$

This is the infinitesimal version of the trivialization described at the beginning of this subsection. There is, however, no well-defined trace function for general elements of $\mathscr{B}_{\text {res }}(H)$ so that this formula makes no sense in the general case.

Now, let $i$ and $j$ be such that $i<j, m_{i}=m_{j}=+\infty$ and $k_{i} \neq k_{j}$. Then we have an element $\Lambda$ in $G L_{\text {res }}(H)$ given by

$$
\begin{aligned}
& \Lambda\left(e_{s_{l}(k)}\right)=e_{s_{l}(k+1)}, \\
& \Lambda\left(e_{s_{J}(k)}\right)=e_{s_{J}(k-1)} \text { if } k>1, \\
& \Lambda\left(e_{s_{l}(k)}\right)=e_{s_{l}(k)} \text { if } l \neq i \text { and } l \neq j . \\
& \Lambda\left(e_{s_{J}(1)}\right)=e_{s_{l}(1)}
\end{aligned}
$$


Now we have that $d \alpha\left(\Lambda, \Lambda^{-1}\right)$ is equal to

$$
k_{i}-k_{j}=k_{i} \text { Trace } \Lambda_{i j}\left(\Lambda^{-1}\right)_{j i}-k_{j} \text { Trace }\left(\Lambda_{j i}^{-1} \Lambda_{i j}\right) \neq 0
$$

In particular $d \alpha$ is a non-trivial Lie algebra 2-cocycle. This implies that also the group 2-cocycle $\alpha$ is non-trivial. For, consider the commuting elements $g_{1}=\exp (t \Lambda)$ and $g_{2}=\exp \left(s \Lambda^{-1}\right)$. In case that $\alpha$ was trivial we would have $\alpha\left(g_{1}, g_{2}\right)=\alpha\left(g_{2}, g_{1}\right)$. However, for sufficiently small $t$ and $s$, the map $(t, s) \mapsto \alpha\left(g_{1}, g_{2}\right)$ is a non-constant holomorphic function, since $d \alpha\left(\Lambda, \Lambda^{-1}\right) \neq 0$. On the other hand one computes directly that for all $i, 1 \leq i \leq m$,

$$
\left(g_{2} g_{1}\right)_{i i}=\left(g_{2}\right)_{i i}\left(g_{1}\right)_{i i} \Longrightarrow \alpha\left(g_{2}, g_{1}\right)=1 \text {. }
$$

This shows that $\alpha\left(g_{1}, g_{2}\right) \neq \alpha\left(g_{2}, g_{1}\right)$ and hence $\alpha$ is a non-trivial 2-cocycle. We summarize this result in a

\section{Theorem 3.4.1.}

(a) The extension $G(\underline{k})$ is alw'ays trivial if there is at most one infinite $m_{i}$.

(b) If there are at least two infinite dimensional components in the basic flag, then $G(\underline{k})$ is trivial if and only if for all $i$ and $j$,

$$
m_{i}=m_{j}=\infty \Longrightarrow k_{i}=k_{j} \text {. }
$$

(c) If $k_{i} \neq k_{j}$ for infinite dimensional $H_{i}$ and $H_{j}$, then the corresponding Lie algebra 2-cocycle for the extension $G(\underline{k})$ is given by

$$
d \alpha(X, Y)=\sum_{i=1}^{m} k_{i} \text { Trace }\left\{\sum_{j \neq i} X_{i j} Y_{j i}-\sum_{j \neq i} Y_{i j} X_{j i}\right\} .
$$

Remark 3.4.2. Consider the case $m=2, m_{1}=m_{2}=\infty, k_{1}=-1$ and $k_{2}=0$ and restrict $d \alpha$ to $\overline{\mathrm{gl}_{(\infty)}} \cap \mathrm{gl}(S)$. Then we have the 2 -cocycle defining the Lie algebra $A_{\infty}$.

3.5. The holomorphic sections of $\boldsymbol{L}(\underline{\boldsymbol{k}})$. Let $\mathfrak{L}(\underline{k})$ denote the space of global holomorphic sections of $L(\underline{k})$. The space $\mathfrak{L}(\underline{k})$ is given the topology of uniform convergence on compact subsets of $\mathfrak{F}^{(0)}$. It becomes then a complete locally convex space, see [16]. Let $\underline{f}: \mathfrak{F}^{(0)} \rightarrow L(\underline{k})$ belong to $\mathfrak{L}(\underline{k})$. Then it can be written as

$$
\underline{f}\left(g \cdot F^{(0)}\right)=[g, f(g)], \quad \text { for all } g \in(\mathfrak{5},
$$

where $f:(\mathfrak{5} \rightarrow \mathbf{C}$ is a holomorphic function satisfying

$$
f(g t)=f(g) \psi_{\underline{k}}(t)^{-1} \quad \text { for all } g \in \mathfrak{G} \text { and all } t \in \mathscr{T}
$$


Thus we can identify $\mathfrak{L}(\underline{k})$ with the space of holomorphic functions on $\mathfrak{b}$ that satisfy this condition. Since each $(g, d)$ in $G$ acts as an analytic diffeomorphism on $\mathfrak{F}^{(0)}$ as well as $L(\underline{k})$, we get a natural action of $G$ on $\mathfrak{L}(\underline{k})$ that corresponds on the functions on $(\mathfrak{G}$ satisfying (13) to

$$
(g, d)(f)\left(g_{1}\right)=f\left(g^{-1} g_{1} d\right), \text { with } g_{1} \in \mathfrak{G} \text { and }(g, d) \in G \text {. }
$$

Let $K_{n}$ be the finite subset of $S$ introduced in subsection 3.2. By restricting the elements of $\mathfrak{L}(\underline{k})$ to $G L\left(H_{K_{n}}\right)$ one obtains a space of holomorphic functions on $G L\left(H_{K_{n}}\right)$ satisfying

$$
f(g t)=f\left(\left(\begin{array}{ccc}
g_{11} & \cdots & g_{1 m} \\
\vdots & & \vdots \\
g_{m 1} & \cdots & g_{m m}
\end{array}\right)\left(\begin{array}{cccc}
t_{11} & \cdots & & t_{1 m} \\
0 & & & \\
\vdots & \ddots & & \\
0 & \cdots & 0 & t_{m m}
\end{array}\right)\right)=\prod_{i=1}^{m} \operatorname{det}\left(t_{i i}\right)^{-k_{2}} f(g),
$$

where $g \in G L\left(H_{K_{n}}\right), t \in G L\left(H_{K_{n}}\right) \cap \mathscr{T}$ and the decomposition of $g$ and $t$ is w.r.t. $H_{K_{n}}=\bigoplus_{i=1}^{m} H_{K_{n} \cap S_{i}}$. The Borel-Weil theorem says that such functions $\neq 0$ exist if and only if $\underline{k}$ satisfies

$$
k_{1} \leq k_{2} \cdots \leq k_{m-1} \leq k_{m}
$$

Since $\mathfrak{F}(\infty)$ is dense in $\mathfrak{F}^{(0)}$, the restriction of some non-zero $f$ in $\mathfrak{L}(\underline{k})$ must be non-zero for a sufficiently large $n$. Hence this condition from the finite dimensional situation is also necessary in this Hilbert context. We will show that it is sufficient too. So we assume from now on that $\underline{k} \in \mathbf{Z}^{m}$ satisfies condition (15).

Before we will construct concrete non-zero elements of $\mathfrak{L}(\underline{k})$, we will first introduce some basic building blocks. If $\Sigma=\left\{\Sigma_{j}\right\}$ belongs to $\mathscr{S}(0)$, then we write

$$
\Sigma(i)=\bigcup_{j \leq i} \Sigma_{j} \quad \text { and } \quad \mathscr{S}_{i}=\{\Sigma(i) \mid \Sigma \in \mathscr{S}(0)\}
$$

Let $\sigma=\sigma_{1} \oplus \cdots \oplus \sigma_{m}$ be an admissible permutation of $S$ corresponding to $\Sigma$. Consider for $g \in(5)$ the operator $\left(\underline{\sigma}_{1} \oplus \cdots \oplus \underline{\sigma}_{i}\right)^{-1} \circ p_{\Sigma(i)} \circ g \mid \underset{j \leq i}{\oplus} H_{j}$ from $\underset{j \leq i}{\oplus} H_{j}$ to itself. It decomposes as

$$
\left(\begin{array}{ccc}
h_{11} & \cdots & h_{1 i} \\
\vdots & \ddots & \vdots \\
h_{i 1} & \cdots & h_{i i}
\end{array}\right) \text {, with } h_{j j}-\operatorname{Id}_{H_{J}} \in \mathcal{N}\left(H_{j}\right), h_{i j} \in \mathscr{H} \mathscr{S}\left(H_{j}, H_{i}\right) \quad \text { for all } j \neq i \text {. }
$$


In particular we can take the determinant of this operator. The function $f_{\Sigma(i)}: \mathbb{G} \rightarrow \mathbf{C}$ defined by

$$
f_{\Sigma(i)}(g)=\operatorname{det}\left(\left(\underline{\sigma}_{1} \oplus \cdots \oplus \underline{\sigma}_{i}\right)^{-1} \circ p_{\Sigma(i)} \circ g \mid \bigoplus_{j \leq i} H_{j}\right)
$$

satisfies for each $t=\left(t_{i j}\right)$ in $\mathscr{T}$ the condition

$$
f_{\Sigma(i)}(g t)=f_{\Sigma(i)}(g) \operatorname{det}\left(t_{11}\right) \cdots \operatorname{det}\left(t_{i i}\right)
$$

In other words $f_{\Sigma(i)}$ belongs to $\mathfrak{I}((-1, \ldots,-1,0, \ldots))$. Now we consider the action of $T(\mathcal{N})$ on such a function $f_{\Sigma(i)}$. Let $g$ be in $\left(5\right.$ and $t=\operatorname{diag}\left(\left\{1+t_{s}\right\}\right)$ in $T(\mathscr{N})$. By definition $f_{\Sigma(i)}\left(t^{-1} g\right)$ is equal to the determinant of the operator

$$
\begin{aligned}
e_{l} \stackrel{g}{\longmapsto} \sum_{r \in S} g_{r l} e_{r} \stackrel{t^{-1}}{\longrightarrow} \sum_{r \in S}\left(1+t_{r}\right)^{-1} g_{r l} e_{r} \\
\stackrel{p H_{\Sigma(1)}}{\longmapsto} \sum_{r \in \Sigma(i)}\left(1+t_{r}\right)^{-1} g_{r l} e_{r} \stackrel{\sigma^{-1}}{\longrightarrow} \sum_{c \in \Sigma(0)(i)}\left(1+t_{\sigma(c)}\right)^{-1} g_{\sigma(c) l} e_{c} .
\end{aligned}
$$

Hence each $f_{\Sigma(i)}$ is an eigenvector for the $T(\mathscr{V})$-action and we have

$$
t \cdot f_{\Sigma(i)}=\prod_{c \in \Sigma^{(0)}(i)}\left(1+t_{\sigma(c)}\right)^{-1} f_{\Sigma(i)} .
$$

If we define the character $\psi_{i}$ of $T(\mathcal{N})$ by

$$
\psi_{i}(t)=\prod_{c \in \Sigma^{(0)}(i)}\left(1+t_{c}\right)^{-1}
$$

then we get in general

$$
t \cdot f_{\Sigma(i)}=\prod_{\substack{\sigma-1(c) \in \Sigma^{(0)}(i) \\ c \in \Sigma^{(0)}(i)}}\left(1+t_{c}\right) \cdot \prod_{\substack{c \in \Sigma^{(0)}(i) \\ \sigma(c) \notin \Sigma^{(0)}(i)}}\left(1+t_{\sigma(c)}\right)^{-1} \cdot \psi_{i}(t) f_{\Sigma(i)} .
$$

Because $\underline{\sigma}$ has index zero, the products in the right hand side are over the same number of elements. Since we have by definition, for each $c_{1} \in \Sigma^{(0)}(i)$ with $\sigma^{-1}\left(c_{1}\right) \notin \Sigma^{(0)}(i)$ and for each $c_{2} \in \Sigma^{(0)}(i)$ with $\sigma\left(c_{2}\right) \notin \Sigma^{(0)}(i)$, that $\chi_{c_{1} \sigma\left(c_{2}\right)} \leq 0$, it is clear that the weight of $f_{\Sigma(i)}$ is less then or equal to $\psi_{i}$. In other words, among the weights of the $\left\{f_{\Sigma(i)}, \Sigma(i) \in \mathscr{S}_{i}\right\}, \psi_{i}$ is maximal. Note that condition (15) on $\underline{k}$ allows you to decompose $\psi_{\underline{k}}$ as follows

$$
\begin{aligned}
\psi_{\underline{k}}(t) & =\prod_{i=1}^{m} \operatorname{det}\left(t_{i i}\right)^{k_{i}} \\
& =\left\{\prod_{i=1}^{m} \operatorname{det}\left(t_{i i}\right)\right\}^{k_{m}} \cdot\left\{\prod_{i=1}^{m-1} \operatorname{det}\left(t_{i i}\right)\right\}^{-k_{m}+k_{m}-1} \cdots\left\{\operatorname{det}\left(t_{11}\right)^{-k_{2}+k_{1}}\right\}
\end{aligned}
$$




$$
=\psi_{m}(t)^{-k_{m}} \cdot \prod_{i<m} \psi_{i}(t)^{k_{i+1}-k_{t}}
$$

Now we choose for each $j, 1 \leq j \leq m-1$ a non-zero homogeneous polynomial $P_{j}$ in the $\left\{f_{\Sigma(j)} \mid \Sigma(j) \in \mathscr{S}_{j}\right\}$ of degree $k_{j+1}-k_{j}$. Let $P_{m}:\left(\mathfrak{G} \rightarrow \mathbf{C}^{*}\right.$ be the function

$$
g \longmapsto \operatorname{det}(g)^{-k_{m}}
$$

From the foregoing formulae it will be clear that $P=\prod_{i=1}^{m} P_{i}$ is a non-zero holomorphic function on $\mathfrak{G}$ that belongs to $\mathfrak{L}(\underline{k})$. If we consider the special choice $\tilde{P}=\prod_{i=1}^{m} \tilde{P}_{i}$

$$
\tilde{P}_{i}=\left(f_{\Sigma^{(0)}(i)}\right)^{k_{1+1}-k_{2}}, \quad 1 \leq i \leq m-1, \quad \tilde{P}_{m}=P_{m},
$$

then the $T(\mathscr{N})$-action on this element of $\mathfrak{I}(\underline{k})$ is given by the character

$$
t \longmapsto \prod_{i=1}^{m-1} \psi_{i}(t)^{k_{t+1}-k_{2}} \cdot \psi_{m}(t)^{-k_{m}}=\psi_{\underline{k}}(t) .
$$

Let $\mathfrak{L}_{f}(\underline{k})$ be the span of the functions $P$ described above. From the $T(\mathcal{N})$-action on the $\left\{f_{\Sigma(i)}\right\}$ one concludes that this is the highest weight occurring in $\mathfrak{L}_{f}(\underline{k})$. If $H$ is finite dimensional then it is known that $\mathfrak{L}_{f}(\underline{k})=\mathfrak{L}(\underline{k})$. By using this and the fact that $\mathfrak{F}(\infty)$ is dense in $\mathfrak{F}^{(0)}$, we get

\section{Theorem 3.5.1.}

(a) The space $\mathfrak{L}(\underline{k})$ is non-zero if and only if $k_{1} \leq \cdots \leq k_{m}$.

(b) The subspace $\mathfrak{L}_{f}(\underline{k})$ lies dense in $\mathfrak{L}(\underline{k})$.

Next we consider the representation of $G$ on $\mathfrak{I}(\underline{k})$. Let $V$ be a closed subspace of $\mathfrak{L}_{f}(\underline{k})$ and let $v$ be a non-zero element of $V$. Then there is an $n$ such that the restriction of $v$ to $G L\left(H_{K_{n}}\right)$ is non-zero. Since the representation of $U\left(H_{K_{n}}\right)$ on the holomorphic functions on $G L\left(H_{K_{n}}\right)$ satisfying (14) is irreducible, we get

$$
\operatorname{span}\left\{u \cdot v \mid u \in U\left(H_{K_{n}}\right)\right\}=\left\{\left.f\right|_{G L\left(H_{K_{n}}\right)} \mid f \in \mathfrak{L}(\underline{k})\right\} .
$$

So, if we define $W$ as the closure of the span of the $\{u \cdot v \mid u \in U(\infty)\}$ then we have for each $v_{1}$ in $V$ a sequence $\left\{w_{n}\right\}$ in $W$ such that $v_{1}\left|G L\left(H_{K_{n}}\right)=w_{n}\right| G L\left(H_{K_{n}}\right)$. Since $U(\infty) \cdot F^{(0)}$ is dense in $\mathfrak{F}^{(0)}$, this implies that $\left\{w_{n}\right\}$ converges to $v_{1}$ and we get that $V=W$. Hence we can say

Theorem 3.5.2. Let $\underline{k}=\left(k_{1}, \ldots, k_{m}\right) \in \mathbf{Z}^{m}$ satisfy $k_{1} \leq \cdots \leq k_{m}$. The repre- 
sentation of $\mathbf{( 5}$ (and hence of $G)$ on $\mathfrak{I}(\underline{k})$ is topologically irreducible.

This Theorem is a generalization of Theorem 10.4.6 in [23].

For each $n \in \mathbf{N}$, we know that the representation of $G L\left(H_{K_{n}}\right)$ on $\left\{f\left|G L\left(H_{K_{n}}\right)\right| f \in \mathfrak{L}(\underline{k})\right\}$ has up to a constant a unique vector that is w.r.t. the $\left\{T(\mathscr{N}) \cap G L\left(H_{K_{n}}\right)\right\}$ action of highest weight

$$
t \longmapsto \prod_{i=1}^{m-1}\left\{\psi_{i}(t)\right\}^{k_{t}+1-k_{2}}(\operatorname{det}(t))^{k_{m}}
$$

In view of the foregoing results we may conclude now for the space $\mathfrak{I}(\underline{k})$ :

Theorem 3.5.3. All $T(\mathscr{N})$-weights $\psi$ occurring in $\mathfrak{L}(\underline{k})$ satisfy.

$$
\psi \leq \prod_{i=1}^{m-1}\left(\psi_{i}\right)^{k_{i+1}-k_{l}} \psi_{m}^{-k_{m}}=\psi_{\underline{k}}
$$

The vector $\tilde{P}$ spans the subspace of vectors with $T\left(\mathcal{N}^{N}\right)$-weight $\psi_{\underline{k}}$.

In view of the results in the Theorems 3.5.2 and 3.5.3, one could call the characters $\psi_{\underline{k}}$ satisfying (15) dominant.

\section{§4. Applications to Integrable Systems}

4.1. A group of commuting flows. In the first subsection we discuss the flows that form the basis of the equations of the multicomponent KP-hierarchy and of the modified equations. Let $H$ be the Hilbert space $L^{2}\left(S^{1}, \mathbf{C}^{r}\right)$ with the usual norm. Let $\left\{f_{t} \mid 1 \leq t \leq r\right\}$ be the standard basis of $\mathbf{C}^{r}$. Then the elements of $H$ can be written as

$$
\sum_{i \in \mathbf{Z}} \sum_{k=1}^{r} \alpha_{i k} f_{k} \lambda^{i}, \quad \text { with } \alpha_{i k} \in \mathbf{C}
$$

The space $H$ is decomposed as $H=H_{1} \oplus H_{2}$ with

$$
H_{1}=\left\{\sum_{i \geq 0} h_{i} \lambda^{i} \mid h_{i} \in \mathbf{C}^{r}\right\} \quad \text { and } \quad H_{2}=H_{1}^{\perp}
$$

The elements $\left\{f_{k} \lambda^{i} \mid 1 \leq k \leq r, i \geq 0\right\}$ are an orthonormal basis of $H_{1}$ and the $\left\{f_{k} \lambda^{i} \mid i<0,1 \leq k \leq r\right\}$ one of $H_{2}$. To get a numbering like in the foregoing section, one defines $e_{k+i r-1}=f_{k} \lambda^{i}$. In the present context it is also convenient to see the matrix $[g]$ of an operator $g$ in $\mathscr{B}_{\text {res }}(H)$ as an $\mathbf{Z} \times \mathbf{Z}$-matrix with entries in $\mathrm{gl}_{r}(\mathbf{C})$, i.e. 


$$
[g]=\left(\begin{array}{cccc}
\ddots & & & \\
& G_{k+1 \ell+1} & G_{k+1 \ell} & \\
& G_{k \ell+1} & G_{k \ell} & \\
& & & \ddots
\end{array}\right) \text {, with } G_{s t} \in \mathrm{gl}_{r}(\mathbf{C}) .
$$

An important operator in $\mathscr{B}_{\text {res }}(H)$ is the multiplication $\Lambda$ on each factor with $\lambda$. It has the matrix [ $\Lambda]$ with $\Lambda_{i i-1}=\operatorname{Id}$ and $\Lambda_{i j}=0$, if $j \neq i-1$. One verifies directly that the centralizer $Z(\Lambda)$ of $\Lambda$ in $\mathscr{B}_{\text {res }}(H)$ consists of all $g$ in $\mathscr{B}_{\text {res }}(H)$ such that the matrix of $g$ looks like

$$
[g]=\left(\begin{array}{ccccc}
\ddots & \ddots & & & \\
\ddots & G_{00} & G_{10} & & \\
& G_{-10} & G_{00} & G_{10} & \ddots \\
& & G_{-10} & G_{00} & \ddots
\end{array}\right) .
$$

Clearly, multiplying with an $A$ from $\operatorname{gl}_{r}(\mathbf{C})$, defines an element of $\mathscr{B}_{\text {res }}(H)$. Let $\mathfrak{h}$ be the diagonal matrices in $\mathrm{gl}_{r}(\mathbf{C})$. It is obvious then that

$$
\left\{\sum_{i \in \mathbf{Z}} H_{i} \Lambda^{i} \in \mathscr{B}_{\text {res }}(H), H_{i} \in \mathfrak{h} \quad \text { for all } i\right\}
$$

is a maximal commutative subalgebra of $\mathscr{B}_{\text {res }}(H)$. The group of commuting flows that we will consider is contained in this algebra and takes care of essentially all independent directions. To be more precise, let $U$ be a connected neighborhood of $S^{1}$ in $\mathrm{C}$ and let $\Gamma(U)$ be the space of all analytic maps $\gamma: U \rightarrow \mathfrak{h}$ such that $\operatorname{det}(\gamma(u)) \neq 0$ for all $u \in U$. In a natural way $\Gamma(U)$ is a group. If $U_{1} \supset U_{2}$ then we get an embedding of $\Gamma\left(U_{1}\right)$ into $\Gamma\left(U_{2}\right)$ by restricting functions to $U_{2}$. We write $\Gamma$ for the inverse limit of the $\{\Gamma(U)\}$. Each $\gamma \in \Gamma$ has a Fourier series

$$
\gamma=\sum_{i \in \mathbf{Z}} \gamma_{i} \lambda^{i}, \gamma_{i} \in \mathfrak{h}
$$

The multiplication with $\gamma$, defines the element $\sum_{i \in \mathbf{Z}} \gamma_{i} \Lambda^{i}$ in $\mathscr{B}_{\text {res }}(H)$. Let $E_{\alpha}$, $1 \leq \alpha \leq r$, be the diagonal matrix in $\operatorname{gl}_{r}(\mathbf{C})$ with $(\alpha, \alpha)$-entry equal to 1 and the other entries equal to zero. At the consideration of the flows from $\Gamma$ on $\mathfrak{F}$ we make use of a decomposition of the elements of $\Gamma$. In $\Gamma$ we consider namely the following subgroups

$$
\begin{aligned}
\Gamma_{+} & =\left\{\gamma \mid \gamma \in \Gamma, \gamma=\exp \left(\sum_{\substack{i>0 \\
1 \leq \alpha \leq r}} t_{i \alpha} E_{\alpha} \lambda_{i}^{i}\right)\right\}, \\
\Gamma_{-} & =\left\{\gamma \mid \gamma=\sum_{j \leq 0} \gamma_{j} \lambda^{j} \in \Gamma\right\} \text { and } \\
\Delta & =\left\{\delta \mid \delta=\operatorname{diag}\left(\lambda^{k_{1}}, \ldots, \lambda^{k_{r}}\right) \text { with } k_{i} \in \mathbf{Z} \text { for all } i\right\} .
\end{aligned}
$$


Then there holds

Lemma 4.1.1. The group $\Gamma$ decomposes as $\Gamma=\Gamma_{+} \Delta \Gamma_{-}$.

This lemma is a direct consequence of the decomposition of holomorphic line bundles over $\mathbf{P}^{1}(\mathbf{C})$, see [11]. As we will see in the third subsection the flows from $\Gamma_{-}$do not contribute to the system. Hence there is no need for a description in coordinates for elements from $\Gamma_{-}$.

4.2. The multicomponent KP-hierarchy. We present here a formal algebraic set-up of this system of equations in which the formulae from the appendix of [27] make sense. It offers one also the possibility to consider these equations from an algebraic point of view. Let $R$ be a complex commutative differential algebra with a collection of commuting derivations $\left\{\partial_{i \alpha} \mid i \geq 1,1 \leq \alpha \leq r\right\}$ of $R$. In the geometric picture $R$ will be an algebra of meromorphic functions on $\Gamma_{+}$and $\partial_{i \alpha}$ will be taking the partial derivative w.r.t. $t_{i \alpha}$. Let $\partial$ be the derivation $\sum_{\alpha=1}^{r} \partial_{1 \alpha}$. The equations of the hierarchy can be formulated conveniently in terms of relations for certain elements from the ring $\mathrm{gl}_{r}(R)\left(\left(\partial, \partial^{-1}\right)\right)$ of pseudo differential operators in $\partial$ with coefficients from $\mathrm{gl}_{r}(R)$. We extend the $\partial_{i \alpha}$ to derivations of $\mathrm{gl}_{r}(R)\left(\left(\partial, \partial^{-1}\right)\right)$ by letting it act coefficient wise on elements of $\mathrm{gl}_{r}(R)$ and on an element $\sum_{j \leq N} p_{j} \partial^{j}$ of $\mathfrak{g l}_{r}(R)\left(\left(\partial, \partial^{-1}\right)\right)$ by

$$
\partial_{i \alpha}\left(\sum_{j \leq N} p_{j} \partial^{j}\right)=\sum_{j \leq N} \partial_{i \alpha}\left(p_{j}\right) \partial^{j}
$$

In the ring $\mathfrak{g l}_{r}(R)\left(\left(\partial, \partial^{-1}\right)\right)$ we denote the differential operator part $\sum_{j \geq 0} p_{j} \partial^{j}$ of $P=\sum_{j} p_{j} \partial^{j}$ by $P_{+}$and we write $P_{-}$for $P-P_{+}$. Let $E_{\alpha}, 1 \leq \alpha \leq r$, be as in the foregoing subsection. In the $\operatorname{ring} \operatorname{gl}_{r}(R)\left(\left(\partial, \partial^{-1}\right)\right)$ we consider elements of the form

$$
L=\partial+\sum_{j>0} t_{j} \partial^{-j} \text { and } U_{\alpha}=E_{\alpha}+\sum_{j>0} u_{\alpha j} \partial^{-j}
$$

Examples of this type of operators can be obtained as follows: take the trivial example $L=\operatorname{Id} \partial=\partial$ and $U_{\alpha}=E_{\alpha}$ and choose some $K=\operatorname{Id}+\sum_{j>0} k_{j} \partial^{-j}$ in $\mathrm{gl}_{\boldsymbol{r}}(R)\left(\left(\partial, \partial^{-1}\right)\right)$. Such a $K$ is invertible and

$$
L=K \partial K^{-1} \quad \text { and } \quad U_{\alpha}=K E_{\alpha} K^{-1}
$$

have the form (16). Following [27], the equations of the multicomponent KP-hierarchy are 


$$
\begin{aligned}
{\left[L, U_{\alpha}\right] } & =\left[U_{\alpha}, U_{\beta}\right]=0 \\
U_{\alpha} U_{\beta} & =\delta_{\alpha \beta} U_{\beta} \\
\partial_{i \alpha}(L) & =\left[\left(L^{i} U_{\alpha}\right)_{+}, L\right]=\left[B_{i \alpha}, L\right] \\
\partial_{i \alpha}\left(U_{\beta}\right) & =\left[B_{i \alpha}, U_{\beta}\right] .
\end{aligned}
$$

The equations (18) and (19) are satisfied by all elements $L$ and $\left\{U_{\alpha}\right\}$ of the form (17). The equations (20) and (21) boil down to non-linear differential equations for the $\left\{u_{\alpha j}\right\}$ and $\left\{\ell_{j}\right\}$. Since all the solutions of the multicomponent KP-hierarchy that we will construct are of the form (17), we merely have to focus on (20) and (21). These last equations can be seen as compatibility conditions for a linear system. This requires the introduction of a $\mathrm{gl}_{r}(R)\left(\left(\partial, \partial^{-1}\right)\right.$ module. Let $M$ consist of the formal products

$$
\left\{\sum_{j=-\infty}^{N} \beta_{j} \lambda^{j}\right\} \exp \left(\sum_{\substack{i>1 \\ 1 \leq \alpha \leq r}} t_{i \alpha} E_{\alpha} \lambda^{i}\right):=\left\{\sum_{j=-\infty}^{N} \beta_{j} \lambda^{j}\right\} g(\hat{\lambda}),
$$

with $\beta_{j} \in \mathfrak{g l}_{r}(R)$. For $\beta \in \mathfrak{g l}_{r}(R)$, the action of $\beta$ on $M$ is defined by

$$
\beta\left\{\sum_{j=-\infty}^{N} \beta_{j} \lambda^{j}\right\} g(\lambda)=\left\{\sum_{j=-\infty}^{N} \beta \beta_{j} \lambda^{j}\right\} g(\lambda) .
$$

The action of $\partial_{i \alpha}$ on $M$ is defined such that it corresponds to "differentiating" this formal product w.r.t. the variable $t_{i \alpha}$, i.e.

$$
\partial_{i \alpha}\left(\left\{\sum_{j} b_{j} \lambda^{j}\right\} g(\lambda)\right)=\left\{\sum_{j} \partial_{i \alpha}\left(b_{j}\right) \lambda^{j}+\sum_{j} b_{j} E_{\alpha} \lambda^{i+j}\right\} g(\lambda)
$$

In particular we see that the action of $\partial$ on $M$

$$
\partial\left\{\sum b_{j} \lambda^{j}\right\} g(\lambda)=\sum\left\{\partial\left(b_{j}\right) \lambda^{j}+\sum b_{j} \lambda^{j+1}\right\} g(\lambda)
$$

is invertible with the inverse $\partial^{-1}$ given by

$$
\partial^{-1}\left\{\sum b_{j} \lambda^{j}\right\} g(\lambda)=\left\{\sum_{i=0}^{\infty} \sum_{j}(-1)^{i} \partial^{i}\left(b_{j}\right) \lambda^{j-i-1}\right\} g(\lambda) .
$$

These actions compose to a $\mathrm{gl}_{r}(R)\left(\left(\partial, \partial^{-1}\right)\right)$-module structure on $M$. In fact, $M$ is a free $\mathrm{gl}_{r}(R)\left(\left(\partial, \partial^{-1}\right)\right.$-module with generator $g(\lambda)$, for, if $P=\Sigma P_{j} \partial^{j} \in$ $\mathrm{gl}_{r}(R)\left(\left(\partial, \partial^{-1}\right)\right)$, then a direct computation shows

$$
P \cdot g(\lambda)=\left\{\sum P_{j} \lambda^{j}\right\} g(\hat{\lambda})
$$

Let $\Delta$ be the subgroup of $\operatorname{gl}_{r}\left(\mathbf{C}\left(\lambda, \lambda^{-1}\right)\right)$ given by

$$
\Delta=\left\{\delta \mid \delta=\operatorname{diag}\left(\lambda^{k_{1}}, \ldots, \lambda^{k_{r}}\right) \text { with }\left(k_{1}, \ldots, k_{r}\right) \in \mathbf{Z}^{r}\right\} .
$$


Take any $\delta=\Sigma d_{j} \lambda^{j}, d_{j} \in \mathfrak{g l} l_{r}(\mathbf{C})$, in $\Delta$. To $\delta$ corresponds the element $\underline{\delta}=\Sigma d_{j} \partial^{j}$ in $\operatorname{gl}_{r}(R)\left(\left(\partial, \partial^{-1}\right)\right)$. Then we have the notion

Definition 4.2.1. A function of type $\delta$ is an element of $\psi$ of $M$ that has the form

$$
\psi=\left\{\left(\operatorname{Id}+\sum_{j<0} \psi_{j} \lambda^{j}\right)\left(\sum_{k} d_{k} \lambda^{k}\right)\right\} g(\lambda)
$$

To any function $\psi$ of type $\delta$ we associate the operator $K_{\psi}$ in $\mathfrak{g l}_{r}(R)\left(\left(\partial, \partial^{-1}\right)\right)$ given by

$$
K_{\psi}=\mathrm{Id}+\sum_{j} \psi_{j} \partial^{j}
$$

Next we assume that we have been given operators $L$ and $\left\{U_{\alpha}\right\}$ of the form (17). Then we introduce the following notion:

Definition 4.2.2. A wavefunction of type $\delta$ for $L$ and the $\left\{U_{\alpha}\right\}$ is a function $\psi$ of type $\delta$ satisfying

(a) $L(\psi)=\lambda \psi$

(b) $U_{\alpha} \psi=\psi E_{\alpha}$

(c) $\partial_{i \alpha}(\psi)=P_{i \alpha} \cdot \psi$ with $P_{i \alpha} \in \mathrm{gl}_{r}(R)[\partial]$.

The first two properties translate respectively into

$$
L=K_{\psi} \partial K_{\psi}^{-1} \text { and } U_{\alpha}=K_{\psi} E_{\alpha} K_{\psi}^{-1} .
$$

Hence $L$ and the $\left\{U_{\alpha}\right\}$ are completely determined by $\psi$. One computes directly that (c) implies $P_{i \alpha}=\left(L^{i} U_{\alpha}\right)_{+}$and by applying the operators $\partial_{i \alpha}$ to the equations (a) and (b) and by substituting (c) one shows

Theorem 4.2.3. If $\psi$ is a wavefunction of type $\delta$, then the operators $K_{\psi} \partial K_{\psi}^{-1}$ and $\left\{K_{\psi} E_{\alpha} K_{\psi}^{-1}\right\}$ satisfy the equations of the multicomponent KP-hierarchy.

The equations from definition 4.2.2 are called a linearization of the system and from theorem 4.2.3 we see that we merely have to show $(c)$ if $L$ and the $\left\{U_{\alpha}\right\}$ are defined by (22).

4.3. The solutions. First we consider the space $H$ and its decomposition as in subsection 4.1. Since $m=2$, all flags in $\mathfrak{F}$ correspond to subspaces $W$ of H. For each $W$ in $\mathfrak{F}$, consider

$\Delta_{W}=\left\{\delta \mid \delta \in \Delta\right.$, there is a $\gamma \in \Gamma_{+}$such that $\gamma^{-1} \delta^{-1} W$ is transversal to $\left.H_{1}\right\}$

The first property of $\Delta_{W}$ is

Lemma 4.3.1. The collection $\Delta_{W}$ is non-empty. 
For each $\delta$ in $\Delta_{W}$ we consider the open subset $\Gamma(\delta, W)$ of $\Gamma_{+}$given by

$$
\Gamma(\delta, W)=\left\{\gamma \mid \in \Gamma_{+}, \gamma^{-1} \delta^{-1} W \text { is transversal to } H_{1}\right\} .
$$

Let $R$ be the ring of analytic functions on $\Gamma(\delta, W)$ and let $\partial_{i \alpha}$ be the derivation of $R$ consisting of partial differentiation w.r.t. $t_{i \alpha}$. Then there holds

\section{Theorem 4.3.2.}

(a) For each $W \in \mathfrak{F}$ and each $\delta \in \Delta_{W}$ there is a unique function $\psi_{W}^{\delta}=$ $\hat{\psi}_{W}^{\delta} \cdot \delta \cdot g(\lambda)$ of type $\delta$, such that $\psi_{W}^{\delta}(\gamma) \in W$ for all $\gamma \in \Gamma(\delta, W)$.

(b) The function $\psi_{W}^{\delta}$ from (a) is a wavefunction of type $\delta$.

For a proof, we refer to [13]. If we write $\psi_{W}^{\delta}=K_{W}^{\delta} \cdot \delta \cdot g(\lambda)$, then we know from theorem 4.2.3 that

$$
L_{W}^{\delta}=K_{W}^{\delta} \partial\left(K_{W}^{\delta}\right)^{-1} \quad \text { and } \quad U_{\alpha, W}^{\delta}=K_{W}^{\delta} E_{\alpha}\left(K_{W}^{\delta}\right)^{-1}
$$

are solutions of the multicomponent KP-hierarchy. The following theorem makes clear why we did not consider the commuting flows from $\Gamma_{-}$. Its proof can also be found in [13].

Theorem 4.3.3. For each $g=\sum_{j \leq 0} \gamma_{j} \lambda^{j}$ in $\Gamma_{-}$, we have $L_{g W}^{\delta}=L_{W}^{\delta}$ and $U_{\alpha, g W}^{\delta}=U_{\alpha, W}^{\delta}$.

Remark 4.3.4. If $r>1$, then $\Delta_{W}$ may contain several elements. If $\delta_{1}$ and $\delta_{2}$ are in $\Delta_{W}$, then the solutions $\left\{L_{W}^{\delta_{1}}, U_{W, \alpha}^{\delta_{1}}\right\}$ and $\left\{L_{W}^{\delta_{2}}, U_{W, \alpha}^{\delta_{2}}\right\}$ are related by so-called differential difference equations that reduce in a specific case to the equations of the Toda-lattice, see [13]. These differential difference equations are a generalization to the KP-level of equations considered in [1].

Also in the multicomponent setting the coefficients of $\hat{\psi}_{W}^{\delta}$ can be expressed in terms of Fredholm determinants related to the line bundle $L((-1,0))$. If $W=g_{1} F^{(0)}$, with $g_{1} \in\left(\mathfrak{5}\right.$, then we define $\tau_{g_{1} \mid H_{1}}: G \rightarrow \mathbf{C}$ by

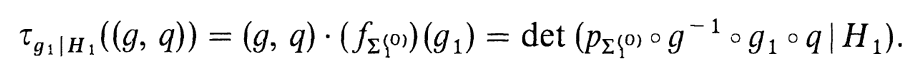

It measures the failure of $G$-equivariance of the section corresponding to $f_{\Sigma(0)}$. If one takes another element $\tilde{g}_{1}$ of $\left(\tilde{\mathfrak{F}}\right.$ with $W=\tilde{g}_{1} F^{(0)}$, then $\tau_{\tilde{g}_{1} \mid H_{1}}$ and $\tau_{g_{1} \mid H_{1}}$ differ by a non-zero constant. In $\Delta$ we consider the elements $\Delta_{i / j}$ given by

$$
\Delta_{i / j}=\operatorname{diag}\left(\ldots \lambda, \ldots, \lambda^{-1}, \ldots\right),
$$

where the $\lambda$-factor stands at the $i$-th place, the $\lambda^{-1}$-factor at the $j$-th place and the resulting factors are equal to 1 .

If $k \in \mathbb{C},|k|>1$, then we still need the element $q_{k}^{(i)}$ from $\Gamma_{+}$given by

$$
q_{k}^{(i)}=\operatorname{diag}\left(\ldots 1,1-\frac{\lambda}{k}, 1, \ldots\right),
$$


where the factor $1-\frac{\lambda}{k}$ stands at the $i$-th place. Then there holds

Theorem 4.3.5. Consider $a W$ in $\mathfrak{F}$ and $a \delta$ in $\Delta_{W}$. Then $\delta^{-1}(W)=g F^{(0)}$, with $g \in \mathbb{6}$ and we have

(a) For all $1 \leq i \leq r$, the $(i, i)$-entry of $\hat{\psi}_{W}^{\delta}$, is the $L^{2}$-boundary value of

$$
k \longmapsto \frac{\tau_{g i H_{1}}\left(\gamma q_{k}^{(i)}\right)}{\tau_{g \mid H_{1}}(\gamma)}
$$

(b) For $j \neq i$, there is a lifting $\tilde{\Delta}_{i / j}$ of $\Delta_{i / j}$ to $G$ such that the $(i, j)$ entry of $\hat{\psi}_{W}^{\delta}$ is the $L^{2}$-boundary value of

$$
k \longmapsto k^{-1} \frac{\tau_{g \mid H_{1}}\left(\gamma, \tilde{\Delta}_{i \mid j} q_{k}^{i}\right)}{\tau_{g \mid H_{1}}(\gamma)} .
$$

This theorem gives a geometric interpretation of formulae, stated in the appendix of [27] and generalizes the one component interpretation given in [25]. For the one component case a representation theoretic interpretation of the $\tau$ depending polynomially of the $\left\{t_{i_{\alpha}}\right\}$ was given in [18]. For a proof of theorem 4.3.5 we refer the reader to [13]. There one can also find more equations that fit in the framework just described.

Next we consider the one component case somewhat more in detail. For convenience we denote the set of independent variables simply as $t=\left\{t_{i} \mid i \geq 1\right\}$ and we see the elements of $R$ as functions in $t$. Further we restrict each $\tau_{g_{1} \mid H_{1}}$ to $\Gamma_{+}$and we write simply $\tau$ or $\tau(t)$. If $k \in \mathbf{Z}$ and $W \in \mathfrak{F}^{(k,-k)}$, then $\Delta_{W}=\left\{\lambda^{-k}\right\}$ and we have exactly one solution $L_{W}^{\lambda^{-k}}$ to the KP-hierarchy. In this way every component of $\mathfrak{F}$ leads to the same bunch of solutions of the KP-hierarchy. Hence, for the construction of solutions, it suffices to consider only one component of $\mathfrak{F}$. The different components are, however, essential for the modified systems as we will see in a moment.

In the one component case the Japanese school, see [17], translates the equations that are satisfied by the wavefunction to equations for the $\tau$-function and these can be written in the so-called bilinear form

$$
\oint \tau\left(\left(t_{i}-\frac{1}{i k^{i}}\right)\right) \tau\left(\left(s_{i}+\frac{1}{i k^{i}}\right)\right) e^{\sum_{i \geq 1}\left(t_{i}-s_{l}\right) k^{2}} d k=0,
$$

for all $\left(t_{i}\right)$ and $\left(s_{i}\right)$ and with $d k$ such that

$$
\oint \frac{d k}{2 \pi i k}=-1
$$

Also this formula can be given a geometric interpretation. For if $W \in \mathfrak{F}^{(0)}$ then one can consider $W^{\perp}$ as an element of the Grassmann manifold corresponding 
to $H=H_{2} \oplus H_{1}$. If $\psi_{W}$ is linked to $\tau$ by theorem 4.3 .5 , then one can show

Theorem 4.3.6. The wavefunction $\psi_{W^{\perp}}$ can be expressed in $\tau$ by

$$
\hat{\psi}_{W^{\perp}}(\lambda)=\lambda^{-1} \frac{\tau\left(\left(t_{i}+\frac{1}{i \lambda^{i}}\right)\right)}{\tau\left(\left(t_{i}\right)\right)}
$$

If we consider instead of $\Gamma_{+}$the group of flows consisting of the adjoints of the elements of $\Gamma_{+}$, then relation (23) boils down to the orthogonality relations for $\psi_{W}$ and $\psi_{W^{\perp}}$.

For $\ell \in \mathbf{Z}$, let $W_{\ell}$ be a general element of $\mathfrak{F}^{(\ell,-\ell)}$. According to the theorems 4.3.2 and 4.3.5 there corresponds a wavefunction of type $\lambda^{\ell}$ to $W_{\ell}$ and a $\tau$-function $\tau_{\ell}$ to $W_{\ell}$. Consider an increasing set of integers $\ell_{1}<\ell_{2} \cdots<\ell_{s}$ and denote it by $\hat{\ell}$. To $\hat{\ell}$ corresponds a decomposition of $H$ by

$$
\begin{aligned}
& H=H_{1} \oplus \cdots \oplus H_{s+1}, \text { where } H_{1}=\left\{\sum_{i \geq \ell_{s}} a_{i} \lambda^{i} \in H\right\}, \\
& H_{j}=\left\{\sum_{\substack{i \geq \ell_{J} \\
i<\ell_{J}+1}} a_{i} \lambda^{i} \in H\right\} \text { for } j, 1 \leq j<s \text { and, } H_{s+1}=\left\{\sum_{i<\ell_{1}} a_{i} \lambda^{i} \in H\right\} .
\end{aligned}
$$

Denote the flagvariety corresponding to this decomposition by $\mathfrak{F}_{\hat{\ell}}$. Then we can describe the elements of $\mathfrak{F}_{\hat{t}}^{(0)}$ in terms of nonlinear equations for the corresponding $\tau$ functions.

Theorem 4.3.7. For $\ell_{1}<\cdots<t_{s}$, let $W_{\ell_{1}}$ be a general elements of $\mathfrak{F}^{\left(\ell_{1},-\ell_{1}\right)}$ and let $\tau_{\ell_{1}}$ be a with $W_{\epsilon_{2}}$ corresponding $\tau$-function. Then the $\left\{W_{t_{1}}\right\}$ determine an element of $\mathfrak{F}_{\hat{\ell}}^{(0)}$ if and only if the $\left\{\tau_{\epsilon_{1}}\right\}$ satisfy the following bilinear equations

$$
\oint \tau_{\ell_{1+1}}\left(\left(t_{j}-\frac{1}{j \lambda^{j}}\right)\right) \tau_{\ell_{1}}\left(\left(s_{j}+\frac{1}{j \lambda^{j}}\right)\right) \lambda^{\ell_{i+1}-\ell_{i}} e^{\Sigma_{j \geq 1}\left(t_{j}-s_{J}\right) \lambda^{j}} d \lambda=0,
$$

for all $\left\{t_{j}\right\}$ and $\left\{s_{j}\right\}$ and for $i, 1 \leq i<s$.

The simplest case of these equations $t_{1}<\ell_{2}$, gives a relation between 2 $\tau$-functions. It has been considered in [17] and is called the $\left(\ell_{2}, \ell_{1}\right)$-modified KP-hierarchy there.

\section{References}

[1] Bergvelt, M. J. and Kroode, A. P. E. ten, Differential-difference AKNS equations and homogeneous Heisenberg algebras, J. Math. Phys., 28 (1987), 302.

[2] Boyer, R. P., Representation Theory of the Hilbert-Lie Group $U(\mathfrak{S})_{2}$, Duke Math. J., 47, No. 2, p. 325-344.

[3] Bourbaki, N., Lie groups and Lie algebras, Chapters 1-3, Springer-Verlag, New York/ 
Berlin, 1989.

[4] - Varietés différentielles et analytiques, Hermann, Paris, 1968.

[5] Carey, A. L. and Ruijsenaars. S. N. M., On fermion gauge groups, current algebras and Kac-Moody algebras, Acta Appl. Math., 10 (1987), 1-86.

[6] Carey, A. L., Hurst, C. A. and O'Brien, D. M., Automorphisms of the canonical anticommutation relations and index theory, J. Funct. Anal., 48 (1982), 360-393.

[7] Date, E., Jimbo, M., Kashiwara, M. and Miwa, T., Transformation groups for soliton equations, Proc. Japan Acad., 57A (1981), 342-7.

[8] Dorfmeister. J. and Neher, E., Banach Jordan pairs and Associated Groups and Manifolds, in preparation.

[9] Dorfmeister, J., Neher, E. and Szmigielski, J., Automorphisms of Banach Manifolds associated with the KP-equation, Quart. J. Math. Oxford, 40 (1989), 161-195.

[10] Faltings, G., Stable G-bundles and projective connections, J. Algebraic. Geom., 2 (1993), $507-568$.

[11] Grothendieck, A., Sur la classification des fibrés holomorphes sur la sphère de Riemann, Am. J. Math., 79 (1957), 121-38.

[12] — La théorie de Fredholm, Bull. Soc. Math. France, 84 (1956), 319-384.

[13] Helminck, G. F. and Post, G. F., The geometry of differential difference equations, Memorandum 999, University of Twente (1991), to appear in Indag. Math.

[14] —, A convergent framework for the multicomponent KP-hierarchy, Trans. Am. Math. Soc., 324, (1991).

[15] - Geometric interpretation of the bilinear equations for the KP-hierarchy, Lett. Math. Phy's., 16 (1988), 359-364.

[16] Herve, M., Analytic and plurisubharmonic functions in finite and infinite dimensional spaces, Lecture Notes in Math., 198 Springer Verlag, Berlin (1971).

[17] Jimbo, M. and Miwa, T., Solitons and infinite dimensional Lie algebras, Publ. RIMS, Kyoto Univ., 19 (1983), 943-1001.

[18] Kac, V. G., Infinite dimensional Lie algebras, Birkhäuser, Boston, 1983.

[19] Kashiwara, M., The flag manifold of Kac-Moody Lie algebra, to appear.

[20] Kuiper, N. H., The homotopy type of the unitary group of Hilbert space, Topology, 3 (1965), 19-30.

[21] Mickelsson, J., Current algebras and groups, Plenum monographs in nonlinear physics.

[22] Peterson, D. H. and Kac, V. G., Infinite flag varieties and conjugacy theorems, Proc. Nat. Acad. Sci. USA, 80 (1983), 1778-1782.

[23] Pressley, A. and Segal, G., Loop groups, Clarendon Press, Oxford. 1986.

[24] Sato, M. and Sato, Y., Soliton equations as dynamical systems on infinite dimensional Grassmann manifolds, Lect. Notes in Num. Appl. Anal., 5 (1982), 259.

[25] Segal, G. B. and Wilson, G., Loop groups and equations of KdV-type, Inst. Hautes Études Sci. Publ. Math., 61 (1985), 5-65.

[26] Shale, D., Linear symmetries of free Boson fields, Trans. Amer. Math. Soc., 103 (1962), 149-167.

[27] Ueno, K. and Takasaki, K., Toda-lattice hierarchy, Adv. Stud. Pure Math., North-Holland. 4 (1984), 1-95. 
